\title{
An extension of Oleinik's inequality for general 1-D scalar conservation laws
}

\author{
Olivier Glass *
}

April 27, 2007

\begin{abstract}
We establish an Oleinik-type inequality concerning $B V$ entropy solutions of general scalar conservation laws in one dimension:

This inequality reads, for $x \leq y$ and $t>0$ :

$$
u_{t}+(f(u))_{x}=0 \text { for }(t, x) \in \mathbb{R}^{+} \times \mathbb{R} .
$$

$$
f^{\prime}(u(t, y))-f^{\prime}(u(t, x)) \leq \frac{y-x}{t}+\sqrt{\left\|\left(f^{\prime \prime}\right)-\right\|_{\infty}\left\|\left(f^{\prime \prime}\right)_{+}\right\|_{\infty}}(T V(u(0, \cdot))-T V(u(t, \cdot))) .
$$

This contains Oleinik's inequality for convex fluxes; in particular, almost convex and almost concave fluxes yield solutions that almost satisfy Oleinik's estimate.

We also show that this inequality is not satisfied in general when one replaces the factor $\sqrt{\left\|\left(f^{\prime \prime}\right)-\right\|_{\infty}\left\|\left(f^{\prime \prime}\right)_{+}\right\|_{\infty}}$ with $\left\|\left(f^{\prime \prime}\right)-\right\|_{\infty}$ or with $\left\|\left(f^{\prime \prime}\right)_{+}\right\|_{\infty}$.
\end{abstract}

\section{Introduction}

We consider a general one-dimensional scalar conservation law:

$$
\partial_{t} u+\partial_{x} f(u)=0 .
$$

Here $f: \mathbb{R} \rightarrow \mathbb{R}$ is a smooth flux function, $u=u(t, x) \in \mathbb{R}$ is the conserved quantity, and $t \in \mathbb{R}^{+}$and $x \in \mathbb{R}$ represent time and position coordinates, respectively.

The well-known result of Kruzkov [12] states that for any $u_{0} \in L^{\infty}(\mathbb{R})$ there exists a unique entropy solution of (1) satisfying

$$
u_{\mid t=0}=u_{0} .
$$

We recall that by entropy solution, we mean a weak solution which moreover satisfies the so-called entropy conditions: for any smooth entropy couple $(\eta, q) \in\left[C^{\infty}(\mathbb{R} ; \mathbb{R})\right]^{2}$ (that is a couple satisfying $q^{\prime}=\eta^{\prime} f^{\prime}$ ) with $\eta$ convex, the following inequality is fulfilled in the sense of measures:

$$
\eta(u)_{t}+q(u)_{x} \leq 0
$$

The classic result of Oleinik [14] states that if $f$ is uniformly strictly convex in the sense that $f^{\prime \prime}(\cdot) \geq \kappa>0$ on $\mathbb{R}$, then any entropy solution of (1) satisfies the following one-sided Lipschitz estimate:

$$
u(t, y)-u(t, x) \leq \frac{y-x}{\kappa t} \text { for any } x \leq y, \text { any } t>0 .
$$

This inequality can be written in a "sharp" form (see Dafermos [5], Hoff [9]): when $f^{\prime \prime}(\cdot) \geq 0$ and moreover $f^{\prime \prime}$ is not identically 0 in a whole interval:

$$
f^{\prime}(u(t, y))-f^{\prime}(u(t, x)) \leq \frac{y-x}{t} \text { for any } x \leq y, \text { any } t>0 .
$$

Several inequalities of this type have been established for non-convex fluxes. The following result has been established by Cheng [3]: for fluxes satisfying the condition that $f^{\prime \prime}$ vanishes at isolated points only, the solutions satisfy:

$$
V^{+}\left[f^{\prime}(u(t, \cdot)) ; I\right] \leq C_{1} \frac{\operatorname{meas}(I)}{t}+C_{2}
$$

for some positive constants $C_{1}$ and $C_{2}$ depending on $f$, where $V^{+}[g ; I]$ designates the positive variation of $g$ over the interval $I$. Jenssen and Sinestrari [11] established the following: if $f$ has exactly one inflexion point and

${ }^{*}$ Université Pierre et Marie Curie-Paris 6, UMR 7598 Laboratoire Jacques-Louis Lions, Paris, F-75005 France. E-mail glass@ann.jussieu.fr 
satisfies an additional condition of "non-flatness" at the inflexion point, then any $B V$ solution satisfies, for some $C>0$ depending on $f$ :

$$
f^{\prime}(u(t, y))-f^{\prime}(u(t, x)) \leq \frac{y-x}{t}+C[T V(u(0, \cdot))-T V(u(t, \cdot))] \text { for } x \leq y \text { and } t>0 .
$$

LeFloch and Trivisa [13] proved the following estimate, which is valid for any flux regardless of the number of inflexion points: for some $C, C^{\prime}>0$ depending on $f$, the $B V$ solutions satisfy

$$
f^{\prime}(u(t, y))-f^{\prime}(u(t, x)) \leq C \frac{y-x}{t}+C^{\prime}[T V(u(0, \cdot))-T V(u(t, \cdot))] \text { for } x \leq y \text { and } t>0,
$$

and the corresponding inequality for $V^{+}\left[f^{\prime}(u(t, \cdot)) ; I\right]$ is valid. Looking more closely at the proof of [13], one can see that the above constants can be described more accurately: for some $C>0$ depending on $f$, one has, for any $\epsilon>0$ and any $B V$ entropy solution $u$ :

$$
f^{\prime}(u(t, y))-f^{\prime}(u(t, x)) \leq(1+\epsilon) \frac{y-x}{t}+\frac{C}{\epsilon}[T V(u(0, \cdot))-T V(u(t, \cdot))] \text { for } x \leq y \text { and } t>0 .
$$

Finally, concerning the spreading of rarefactions waves for solutions of systems of conservation laws —a problem which we do not address here- let us also mention [2] (for genuinely nonlinear systems) and [13] (for general systems), and references therein.

The goal of this paper is to improve results such as (6), (7), (8) and (9) for general flux functions $f$, in a way that extends Oleinik's original inequality (4)-(5) for convex fluxes.

Precisely, we prove the following result:

Theorem 1. Consider $f \in C^{\infty}(\mathbb{R} ; \mathbb{R})$. For any $u_{0} \in L^{\infty}(\mathbb{R}) \cap B V_{\text {loc }}(\mathbb{R})$, the unique entropy solution of (1)-(2) satisfies:

$$
\begin{aligned}
& f^{\prime}(u(t, y))-f^{\prime}(u(t, x)) \leq \frac{y-x}{t}+\sqrt{\left\|\left(f^{\prime \prime}\right)-\right\|_{\infty}\left\|\left(f^{\prime \prime}\right)_{+}\right\|_{\infty}}[T V(u(0, \cdot))-T V(u(t, \cdot))] \\
& \qquad \text { for } x \leq y \text { and } t>0
\end{aligned}
$$

where the $L^{\infty}$ norms are considered in $L^{\infty}\left(\left[-\left\|u_{0}\right\|_{\infty},\left\|u_{0}\right\|_{\infty}\right]\right)$.

In (10), we used the notation for the negative part $x_{-}:=\max (-x, 0)$ and for the positive part $x_{+}:=\max (x, 0)$ (in such a way that $x=x_{+}-x_{-}$). In the whole sequel, we will systematically use the notation $\left\|f^{\prime \prime}\right\|_{\infty}$ and its variants $\left\|\left(f^{\prime \prime}\right)_{-}\right\|_{\infty}$, etc. for $\left\|f^{\prime \prime}\right\|_{L^{\infty}\left(\left[-\left\|u_{0}\right\|_{\infty},\left\|u_{0}\right\|_{\infty}\right]\right)},\left\|\left(f^{\prime \prime}\right)_{-}\right\|_{L^{\infty}\left(\left[-\left\|u_{0}\right\|_{\infty},\left\|u_{0}\right\|_{\infty}\right]\right)}$, etc. Actually, in the whole paper, all the states considered will systematically belong to $\left[-\left\|u_{0}\right\|_{\infty},\left\|u_{0}\right\|_{\infty}\right]$ even when not explicitly mentioned. Finally, we choose as a normalization to consider the solutions $u$ as left-continuous.

Theorem 1 will be shown as a direct consequence of these two joined results:

Proposition 1. Under the assumptions of Theorem 1, the unique entropy solution of (1)-(2) satisfies:

$$
f^{\prime}(u(t, y))-f^{\prime}(u(t, x)) \leq \frac{y-x}{t}+\sqrt{\left\|\left(f^{\prime \prime}\right)-\right\|_{\infty}\left\|f^{\prime \prime}\right\|_{\infty}}[T V(u(0, \cdot))-T V(u(t, \cdot))] \text { for } x \leq y \text { and } t>0 .
$$

Proposition 2. Under the assumptions of Theorem 1, the unique entropy solution of (1)-(2) satisfies:

$$
f^{\prime}(u(t, y))-f^{\prime}(u(t, x)) \leq \frac{y-x}{t}+\sqrt{\left\|\left(f^{\prime \prime}\right)_{+}\right\|_{\infty}\left\|f^{\prime \prime}\right\|_{\infty}}[T V(u(0, \cdot))-T V(u(t, \cdot))] \text { for } x \leq y \text { and } t>0 .
$$

In fact, Proposition 2 can be deduced directly from Proposition 1; hence the main part of this paper is devoted to the proof of Proposition 1. However, as will appear, in some sense we estimate different parts of the solution when obtaining (11) and (12), which somehow explains that one cannot prove these two results simultaneously. This is explained in Paragraph 6.1.

As we will see, Theorem 1 can be slightly strengthened as follows.

Theorem 1'. Under the assumptions of Theorem 1, the unique entropy solution of (1)-(2) satisfies, for any interval I:

$$
V^{+}\left[f^{\prime}(u(t, \cdot)) ; I\right] \leq \frac{\operatorname{meas}(I)}{t}+\sqrt{\left\|\left(f^{\prime \prime}\right)_{-}\right\|_{\infty}\left\|\left(f^{\prime \prime}\right)_{+}\right\|_{\infty}}[T V(u(0, \cdot))-T V(u(t, \cdot))] \text { for } t>0 .
$$


Remark 1. In both Theorems 1 and 1', the constant $\sqrt{\left\|\left(f^{\prime \prime}\right)_{-}\right\|_{\infty}\left\|\left(f^{\prime \prime}\right)_{+}\right\|_{\infty}}$ is not optimal and in fact could be replaced by the slightly better constant

$$
\begin{aligned}
\min \left(\max \left[\frac{\left\|\left(f^{\prime \prime}\right)-\right\|_{\infty}}{2}, \sqrt{\left\|\left(f^{\prime \prime}\right)_{-}\right\|_{\infty}\left(\left\|\left(f^{\prime \prime}\right)_{-}\right\|_{\infty}+\left\|\left(f^{\prime \prime}\right)_{+}\right\|_{\infty}\right)}-\left\|\left(f^{\prime \prime}\right)_{-}\right\|_{\infty}\right]\right. \\
\left.\max \left[\frac{\left\|\left(f^{\prime \prime}\right)_{+}\right\|_{\infty}}{2}, \sqrt{\left\|\left(f^{\prime \prime}\right)_{+}\right\|_{\infty}\left(\left\|\left(f^{\prime \prime}\right)_{+}\right\|_{\infty}+\left\|\left(f^{\prime \prime}\right)_{-}\right\|_{\infty}\right)}-\left\|\left(f^{\prime \prime}\right)_{+}\right\|_{\infty}\right]\right) .
\end{aligned}
$$

This is explained in Paragraph 6.4.

Note the following feature of Theorem 1: it includes Oleinik's inequality in the sense that it coincides with it when the flux is convex (or concave). In particular, the above form of the inequality involves that if the flux is "almost" convex, then the corresponding solutions "almost" satisfy Oleinik's inequality. Now we state a result which is weaker than Theorem 1 in the sense that it does not satisfy this property. The point is that the proof of this inequality is much simpler. We think that the important simplifications that can be made in this case may motivate the objects which we introduce in the course of the proof of Proposition 1. This is developed in Paragraph 6.2.

Proposition 3. Under the assumptions of Theorem 1, the unique entropy solution of (1)-(2) satisfies:

$$
f^{\prime}(u(t, y))-f^{\prime}(u(t, x)) \leq \frac{y-x}{t}+\frac{\left\|f^{\prime \prime}\right\|_{\infty}}{2}[T V(u(0, \cdot))-T V(u(t, \cdot))] \text { for } x \leq y \text { and } t>0
$$

Finally, we will show the following result.

Theorem 2. The conclusions of Theorem 1 are false in general when the factor $\sqrt{\left\|\left(f^{\prime \prime}\right)_{+}\right\|_{\infty} \|_{\left(f^{\prime \prime}\right)_{-} \|_{\infty}}}$ is replaced with $\left\|\left(f^{\prime \prime}\right)_{-}\right\|_{\infty}$ or with $\left\|\left(f^{\prime \prime}\right)_{+}\right\|_{\infty}$ (or even with any factor of the type $\mathcal{O}\left(\left\|\left(f^{\prime \prime}\right)_{-}\right\|_{\infty}\right)$ or $\left.\mathcal{O}\left(\left\|\left(f^{\prime \prime}\right)_{+}\right\|_{\infty}\right)\right)_{\text {. }}$

The cited results in [3], [11] and [13] rely on the theory of generalized characteristics due to Dafermos [6]. Here we rely on a process of selection of binary trees inside front-tracking approximations of the solution of (1)-(2). We recall that the front-tracking method was introduced by Dafermos as well [4]; here we use a version of the algorithm which is an adaptation of a method due to LeFloch and the author [8] (for general hyperbolic systems of conservation laws). This method relies on the so-called inner speed variation estimates (also introduced in [8]), which are central in the proof here.

The paper is organized as follows. In Section 2, we introduce the front-tracking method that we use to construct solutions of (1). In Section 3, we introduce some central material needed in the proof. In Section 4, we establish Proposition 1. In Section 5 we prove the technical results announced in earlier sections. Finally, in Section 6, we prove Proposition 2; we also explain the wide simplifications that could be made to establish Proposition 3; then we prove Theorem $1^{\prime}$ and justify Remark 1; finally we prove Theorem 2.

\section{Front-tracking algorithm}

In this section, we construct particular front-tracking approximations of solutions of (1); this is a remodeling of the algorithm of [8] (note that the scalar case is much simpler), in an adequate way for the sequel. This is done for $u_{0} \in L^{1}(\mathbb{R}) \cap B V(\mathbb{R})$.

Recall that front-tracking approximations are functions which are piecewise constant over a polygonal subdivision of $\mathbb{R}^{+} \times \mathbb{R}$. We will call each of the line segments of this subdivision fronts, each of its vertices interaction points; given an interaction point, the outgoing fronts (resp. the incoming fronts) will refer to the fronts touching the interaction point, located after it (resp. before it) in time. When we follow through time several fronts connected by interaction points, we will call the resulting piecewise affine curve a front path.

\subsection{Riemann solvers}

The front-tracking method consists in putting together suitable approximations of the solutions of Riemann problems. Let us describe the classical solution of the Riemann problem as well as the approximations that we will use.

Exact Riemann solver. We refer for instance to [7] for this part. The Riemann problem consists in finding a self-similar entropy solution $u(t, x)=\bar{u}(x / t)$ of equation (1) for the initial condition:

$$
u(0, x)=\left\{\begin{array}{l}
u_{l} \text { for } x<0, \\
u_{r} \text { for } x>0,
\end{array}\right.
$$


where $u_{l}$ and $u_{r}$ are constants. Recall that for a single discontinuity

$$
u(t, x)=\left\{\begin{array}{l}
u_{-} \text {for } x<c t \\
u_{+} \text {for } x>c t
\end{array}\right.
$$

equation (1) is equivalent to the Rankine-Hugoniot relation

$$
\frac{f\left(u_{+}\right)-f\left(u_{-}\right)}{u_{+}-u_{-}}=c
$$

and the entropy conditions are equivalent to Oleinik's E-condition:

$$
\frac{f(u)-f\left(u_{-}\right)}{u-u_{-}} \geq \frac{f\left(u_{+}\right)-f\left(u_{-}\right)}{u_{+}-u_{-}} \text {for } u \text { between } u_{-} \text {and } u_{+} .
$$

Classically (see e.g. $[7, \S 14.1]$ ), the Riemann problem for scalar conservation laws is solved as follows. Given $a \leq b$, we denote by $\operatorname{conv}_{[a, b]} f\left(\right.$ resp. $\left.\operatorname{conc}_{[a, b]} f\right)$ the convex (resp. concave) hull of $f$ on $[a, b]$. We introduce the function $g$ in the following way:

- if $u_{l}<u_{r}$, we fix $g:=\left(\operatorname{conv}_{\left[u_{l}, u_{r}\right]} f\right)$,

- if $u_{l}>u_{r}$, we fix $g:=\left(\operatorname{conc}_{\left[u_{r}, u_{l}\right]} f\right)$.

If $u_{l}<u_{r}$ (resp. if $u_{r}<u_{l}$ ), the function $g^{\prime}$ in non-decreasing on $\left[u_{l} ; u_{r}\right]$ (resp. non-increasing on $\left[u_{r}, u_{l}\right]$ ); consequently, in both cases the function $g^{\prime}$ has an inverse, which is possibly discontinuous: let us choose to take it left-continuous in the first case and right-continuous in the second case.

Now the wave fan is given by

$$
u(t, x)=\left\{\begin{array}{l}
u_{l} \text { for } \frac{x}{t}<g^{\prime}\left(u_{l}\right), \\
{\left[g^{\prime}\right]^{-1}(x / t) \text { for } g^{\prime}\left(u_{l}\right)<\frac{x}{t}<g^{\prime}\left(u_{r}\right),} \\
u_{r} \text { for } \frac{x}{t}>g^{\prime}\left(u_{r}\right) .
\end{array}\right.
$$

In the sequel, we will denote by $\bar{\lambda}^{\text {min }}\left(u_{l} ; u_{r}\right)$ and $\bar{\lambda}^{\max }\left(u_{l} ; u_{r}\right)$ the lowest and highest wave speeds in the Riemann problem $\left(u_{l} ; u_{r}\right)$, respectively. Precisely:

$$
\bar{\lambda}^{\min }\left(u_{l} ; u_{r}\right):=g^{\prime}\left(u_{l}\right) \text { and } \bar{\lambda}^{\max }\left(u_{l} ; u_{r}\right):=g^{\prime}\left(u_{r}\right) .
$$

As in [8], this allows to introduce the inner speed variation (isv in short) as the following value:

$$
\vartheta\left(u_{l} ; u_{r}\right):=\bar{\lambda}^{\max }\left(u_{l} ; u_{r}\right)-\bar{\lambda}^{\min }\left(u_{l} ; u_{r}\right) .
$$

Approximate Riemann solvers. We introduce two kinds of approximate solvers. The principle is the following: these solvers regroup parts of the outgoing wave fan as "wave packets" and make these packets travel as a single discontinuity (a front), whose (single) speed is the lowest wave speed in the packet. The two solvers are the following.

- The approximate solver using the splitting strategy, with precision $\varepsilon$, generates the following piecewise constant approximation of (17). We introduce $N$ as

$$
N:=\left\lceil\frac{\bar{\lambda}^{\max }\left(u_{l} ; u_{r}\right)-\bar{\lambda}^{\min }\left(u_{l} ; u_{r}\right)}{\varepsilon}\right\rceil,
$$

where $\lceil\cdot\rceil$ is the upper integer part. We introduce the intermediate speeds $\lambda_{0} \ldots \lambda_{N}$ :

$$
\begin{aligned}
\lambda_{0}:=\bar{\lambda}^{\min }\left(u_{l} ; u_{r}\right), \quad \lambda_{1}:=\bar{\lambda}^{\max }\left(u_{l} ; u_{r}\right), & \text { if } N=1, \\
\lambda_{i}:=\bar{\lambda}^{\min }\left(u_{l} ; u_{r}\right)+\frac{i}{2}\left(\bar{\lambda}^{\max }\left(u_{l} ; u_{r}\right)-\bar{\lambda}^{\min }\left(u_{l} ; u_{r}\right)\right), 0 \leq i \leq 2 & \text { if } N=2, \\
\lambda_{0}:=\bar{\lambda}^{\min }\left(u_{l} ; u_{r}\right), & \\
\lambda_{1}:=\bar{\lambda}^{\min }\left(u_{l} ; u_{r}\right)+\varepsilon, & \text { if } N \geq 3 . \\
\lambda_{i}:=\lambda_{1}+\frac{i-1}{N-2}\left(\lambda_{N-1}-\lambda_{1}\right), \quad 1 \leq i \leq N-1, & \\
\lambda_{N-1}:=\bar{\lambda}^{\max }\left(u_{l} ; u_{r}\right)-\varepsilon, & \\
\lambda_{N}:=\bar{\lambda}^{\max }\left(u_{l} ; u_{r}\right), &
\end{aligned}
$$


(See Remark 3 below concerning this particular choice for cutting the Riemann wave fan.) We introduce the corresponding intermediate states:

$$
v_{i}:=\left[g^{\prime}\right]^{-1}\left(\lambda_{i}\right) \text { for } i=0 \ldots N,
$$

or, in other words,

$$
\begin{array}{ll}
v_{i}:=\min \left\{u \in\left[u_{l}, u_{r}\right],\right. & \left.g^{\prime}(u)=\lambda_{i}\right\} \text { if } u_{l}<u_{r}, \\
v_{i}:=\max \left\{u \in\left[u_{r}, u_{l}\right], \quad g^{\prime}(u)=\lambda_{i}\right\} \text { if } u_{r}<u_{l} .
\end{array}
$$

The solver with splitting strategy generates the following approximation of (17):

$$
u(t, x)=\left\{\begin{array}{l}
u_{l}=v_{0} \text { for } x / t<\lambda_{0}, \\
v_{i} \text { for } \lambda_{i-1}<x / t<\lambda_{i}, \quad i=1 \ldots N-1, \\
u_{r} \text { for } x / t>\lambda_{N-1} .
\end{array}\right.
$$

Hence, there are $N$ outgoing fronts, separating $N+1$ states (including $u_{l}$ and $u_{r}$ ).

- The approximate solver using the no-splitting strategy, generates the following (cruder) approximation of the Riemann problem $\left(u_{l}, u_{r}\right)$ :

$$
u(t, x)=\left\{\begin{array}{l}
u_{l} \text { for } x / t<\bar{\lambda}^{\min }\left(u_{l} ; u_{r}\right), \\
u_{r} \text { for } x / t>\bar{\lambda}^{\min }\left(u_{l} ; u_{r}\right) .
\end{array}\right.
$$

In other terms, the Riemann fan is approximated by a single front traveling at its lowest speed.

Remark 2. Note that in the above constructions, each intermediate state $v$ in $\left\{v_{0}, v_{1}, \ldots, v_{N}, u_{r}\right\}$ satisfies $f(v)=\operatorname{conv}_{\left[u_{l} ; u_{r}\right]} f(v)$ if $u_{l}<u_{r}$ (respectively $f(v)=\operatorname{conc}_{\left[u_{r} ; u_{l}\right]} f(v)$ if $u_{l}>u_{r}$ ). It follows that each front separating $u_{-}$and $u_{+}$which has been constructed by one of the above solvers satisfies

$$
\left[\operatorname{conv}_{\left[u_{l} ; u_{r}\right]} f\right]_{\mid\left[u_{-} ; u_{+}\right]}=\operatorname{conv}_{\left[u_{-} ; u_{+}\right]} f \quad\left(\operatorname{resp} .\left[\operatorname{conc}_{\left[u_{r} ; u_{l}\right]} f\right]_{\mid\left[u_{+} ; u_{-}\right]}=\operatorname{conc}_{\left[u_{+} ; u_{-}\right]} f\right) .
$$

As a consequence, each front travels at speed $\bar{\lambda}^{\min }\left(u_{-} ; u_{+}\right)$. It also follows that any outgoing front in the solver using the splitting strategy has an inner speed variation $\vartheta\left(u_{-} ; u_{+}\right)$no more than $\varepsilon$ (and exactly $\varepsilon$ for the leftmost and rightmost ones in (19)).

Remark 3. The convention (19) is different from the one in [8], where the choice for cutting the Riemann wave fan is to take

$$
\lambda_{i}:=\bar{\lambda}^{\min }\left(u_{l} ; u_{r}\right)+\frac{i}{N}\left(\bar{\lambda}^{\max }\left(u_{l} ; u_{r}\right)-\bar{\lambda}^{\min }\left(u_{l} ; u_{r}\right)\right) .
$$

In other words, in (19) we choose to "charge" a little bit more the leftmost and the rightmost fronts. This has absolutely no importance in this section (it only matters here that all fronts travel approximately at the correct speed and have an inner speed variation no more than $\varepsilon$ ), but it will be technically easier to handle (19) than (22) in Section 4.

\subsection{Front-tracking approximations.}

Now we construct a sequence of front-tracking approximations of solutions of the Cauchy problem (1) with initial condition

$$
u(0, x)=u_{0}(x) \text { in } \mathbb{R},
$$

where $u_{0}$ is given in $B V(\mathbb{R}) \cap L^{1}(\mathbb{R})$. Let us describe the algorithm.

1. First, for each $\varepsilon>0$, we take a piecewise constant approximation $u_{0, \varepsilon}$ of $u_{0}$, with compact support, satisfying:

$$
\begin{aligned}
& \inf _{\mathbb{R}} u_{0} \leq u_{0, \varepsilon} \leq \sup _{\mathbb{R}} u_{0}, \\
& T V\left(u_{0}\right)-\varepsilon \leq T V\left(u_{0, \varepsilon}\right) \leq T V\left(u_{0}\right) \\
& \left\|u_{0, \varepsilon}-u_{0}\right\|_{L^{1}(\mathbb{R})} \leq \varepsilon
\end{aligned}
$$

2. At each jump point $x$ of $u_{0, \varepsilon}$, we approximately solve the Riemann problem $u_{0, \varepsilon}\left(x_{-} ; x_{+}\right)$by means of the approximate solver with splitting strategy, with precision $\varepsilon$.

3. We let the fronts evolve at their constant speeds until two fronts meet: hence we get a piecewise constant function $u_{\varepsilon}$ defined for small $t>0$.

4. The function $u_{\varepsilon}$ is extended over an interaction point $P$ (where two fronts meet) according to the following procedure. Suppose that exactly two fronts interact, and call $u_{l}, u_{m}$ and $u_{r}$ the left, middle and right states respectively; then: 
- if $\vartheta\left(u_{l} ; u_{r}\right)>2 \varepsilon$, extend $u_{\varepsilon}$ beyond the interaction point by using the approximate solver with splitting strategy;

- else, use the approximate solver with no-splitting strategy.

Additional convention. If needed, we modify the speeds of an amount of $\varepsilon$ at most in order that at each time, at most one interaction takes place, and this interaction involves exactly two fronts. Moreover, this can be done in such a way that two fronts that meet would have met regardless of this modification and that the order of the fronts (according to the variable $x$ ) is never changed. To get this property, apply the following procedure: if for some time there are at least three fronts concerned with interactions, simply accelerate the involved front which is the most on the left, by a suitably small quantity. This advances the time of the leftmost interaction. In the sequel, we will refer to this additional amount of speed as the additional displacement.

The goal of the above strategy is to construct an approximation where all fronts have a uniformly small inner speed variation. For this reason, fronts having an inner speed variation no more than $\varepsilon$ are considered as completely satisfactory, while fronts having an inner speed variation larger than $2 \varepsilon$ are considered as unacceptable and have to be split (in pieces having an inner speed variation no more than $\varepsilon$ ). Hence as long as the approximation is defined, one can already affirm that

$$
\text { for any front } \alpha \text { in the approximation, } \vartheta(\alpha) \leq 2 \varepsilon \text {, }
$$

where $\vartheta(\alpha):=\vartheta\left(u_{-}^{\alpha}, u_{+}^{\alpha}\right), u_{-}^{\alpha}$ and $u_{+}^{\alpha}$ being the states on the left and on the right of $\alpha$, respectively.

\subsection{Inner speed variation estimates}

In order to prove that the above algorithm is well defined, we will have to estimate the evolution of the inner speed variation across interactions $\left(u_{l}, u_{m}\right)+\left(u_{m}, u_{r}\right) \rightarrow\left(u_{l}, u_{r}\right)$ where $u_{l}, u_{m}$ and $u_{r}$ are the left, middle and right states respectively. From now, we will systematically use this notations for the three incoming states at an interaction point. Sometimes, we will use the notations $u_{l}^{P}, u_{m}^{P}$ and $u_{r}^{P}$ to specify that they correspond to the interaction point $P$. An interaction satisfying $\left(u_{m}-u_{l}\right)\left(u_{r}-u_{m}\right) \geq 0$ is called monotone; it is called nonmonotone otherwise. Also, at an interaction point, we will call the larger front, the incoming front maximizing the difference between the states that it separates, in absolute value.

We have the following estimates:

Proposition 4 (Estimates on the inner speed variation). Across an interaction, one has for some $C_{i}>0$ :

- Monotone case: $\left(u_{l}<u_{m}<u_{r}\right.$ or $\left.u_{r}<u_{m}<u_{l}\right)$ :

$$
\vartheta\left(u_{l}, u_{r}\right) \leq \max \left(\vartheta\left(u_{l}, u_{m}\right), \vartheta\left(u_{m}, u_{r}\right)\right)+\left(\bar{\lambda}^{\min }\left(u_{m} ; u_{r}\right)-\bar{\lambda}^{\min }\left(u_{l} ; u_{m}\right)\right)_{+} .
$$

- Non-monotone case 1 - larger front on the left: $\left(u_{m}<u_{r}<u_{l}, u_{l}<u_{r}<u_{m}\right)$ :

$$
\vartheta\left(u_{l}, u_{r}\right)-\vartheta\left(u_{l} ; u_{m}\right) \leq C_{i}\left|u_{r}-u_{m}\right| .
$$

- Non-monotone case 2 - larger front on the right: $\left(u_{m}<u_{l}<u_{r}, u_{r}<u_{l}<u_{m}\right)$ :

$$
\vartheta\left(u_{l}, u_{r}\right)-\vartheta\left(u_{m} ; u_{r}\right) \leq C_{i}\left|u_{l}-u_{m}\right| \text {. }
$$

Moreover, one can take in (27) and (28):

$$
C_{i}=\sqrt{\left\|\left(f^{\prime \prime}\right)-\right\|_{\infty}\left\|f^{\prime \prime}\right\|_{\infty}} .
$$

A version for systems of such inequalities has been established in [8]. Since the above version is more precise (but it works only for for scalar equations!), we will establish Proposition 4 in Section 5. Note that the precise value of the constant $C_{i}$ in (29) is useless in this section. However, as easily guessed, it will be important in the proof of Proposition 1. 


\subsection{Finite number of fronts and interaction points.}

Let us show that the total number of fronts and the number of interaction points are finite in the above construction. This will prove that the algorithm can be continued for all times.

Call $N(t)$ the total number of fronts at time $t$, and introduce the following value (which somehow measures the total "focusing" of the approximation):

$$
\Theta^{\varepsilon}(t):=\sum_{\alpha \text { front }} \frac{(\vartheta(\alpha)-\varepsilon)_{+}}{\varepsilon} .
$$

That the numbers of fronts and interaction points remain finite is a direct consequence of the following lemma (compare with [8, Lemma 5.1]).

Lemma 1. The functional

$$
\mathcal{I}(t):=\frac{2 C_{i}}{\varepsilon} T V\left(u_{\varepsilon}(t)\right)+N(t)+2 \Theta^{\varepsilon}(t),
$$

is non-increasing, and moreover $\mathcal{I}\left(t^{+}\right)-\mathcal{I}\left(t^{-}\right) \leq-1$ across an interaction time.

Proof of Lemma 1. Of course $\mathcal{I}$ is constant between interaction times, and we only have to prove the second assertion. This is discussed according to the type of interaction under view; call $P$ the interaction point. We denote by $\Delta T V\left(u_{\varepsilon}\right)(t)$ the dissipation of total variation across time $t$, counted positively, i.e. $\Delta T V\left(u_{\varepsilon}\right)(t):=$ $\left[T V\left(u_{\varepsilon}\right)\right]\left(t^{-}\right)-\left[T V\left(u_{\varepsilon}\right)\right]\left(t^{+}\right)$. (Recall that in all cases, $T V\left(u_{\varepsilon}\right)$ does not increase.)

- Monotone interactions: in that case, as the two fronts meet (and would have met regardless of the modification of the speeds), we deduce that

$$
\left(\bar{\lambda}^{\min }\left(u_{m}^{P} ; u_{r}^{P}\right)-\bar{\lambda}^{\min }\left(u_{l}^{P} ; u_{m}^{P}\right)\right)_{+}=0
$$

Hence it follows from (26) that

$$
\vartheta\left(u_{l}^{P}, u_{r}^{P}\right) \leq \max \left(\vartheta\left(u_{l}^{P}, u_{m}^{P}\right), \vartheta\left(u_{m}^{P}, u_{r}^{P}\right)\right) .
$$

Hence, the no-splitting strategy is always used at a monotone interaction point. This fact will be frequently used in the sequel. Consequently in this situation none of the three terms of $\mathcal{I}$ increases. And the second term satisfies $N\left(t^{+}\right)-N\left(t^{-}\right)=-1$.

- Non-monotone interactions where the no-splitting strategy is used: in that case it follows from (27)-(28) that

$$
\Theta^{\varepsilon}\left(t^{+}\right)-\Theta^{\varepsilon}\left(t^{-}\right) \leq \frac{C_{i}}{\varepsilon} \Delta T V\left(u_{\varepsilon}\right)(t) .
$$

As in that case, we still have $N\left(t^{+}\right)-N\left(t^{-}\right)=-1$, the claim follows.

- Non-monotone interactions where the splitting strategy is used: in that case, it follows from the construction that

$$
N\left(t^{+}\right)-N\left(t^{-}\right) \leq \frac{\vartheta\left(u_{l}^{P} ; u_{r}^{P}\right)}{\varepsilon}-1 .
$$

The fact that we used this solver means that $\vartheta\left(u_{l}^{P} ; u_{r}^{P}\right)>2 \varepsilon$. Denote $\underline{\vartheta}$ the inner speed variation of the larger incoming front. It follows from Proposition 4 that

$$
2 \varepsilon \leq \vartheta\left(u_{l}^{P} ; u_{r}^{P}\right) \leq \underline{\vartheta}+C_{i} \Delta T V\left(u_{\varepsilon}\right)(t)
$$

hence

It follows that

$$
\underline{\vartheta}+C_{i} \Delta T V\left(u_{\varepsilon}\right)(t) \geq \frac{2 \varepsilon+\vartheta\left(u_{l}^{P} ; u_{r}^{P}\right)}{2}
$$

$$
2(\underline{\vartheta}-\varepsilon)_{+} \geq 2(\underline{\vartheta}-\varepsilon) \geq \vartheta\left(u_{l}^{P} ; u_{r}^{P}\right)-2 C_{i} \Delta T V\left(u_{\varepsilon}\right)(t) .
$$

Since it follows from the construction that each outgoing front has an inner speed variation less than or equal to $\varepsilon$, we have that

$$
\Theta^{\varepsilon}\left(t^{+}\right)-\Theta^{\varepsilon}\left(t^{-}\right) \leq-\frac{(\underline{\vartheta}-\varepsilon)_{+}}{\varepsilon}
$$

and the result follows. 


\subsection{Limit of the front-tracking sequence.}

The two following classical bounds result easily from the construction: the total variation of $u_{\varepsilon}$ is uniformly bounded (as a non-increasing in time functional):

$$
T V\left(u_{\varepsilon}(t,, \cdot)\right) \leq T V\left(u_{\varepsilon}(0, \cdot)\right) \leq T V\left(u_{0}\right),
$$

and one has the Lipschitz in time estimate (due to the finite speed of propagation)

$$
\left\|u_{\varepsilon}(t)-u_{\varepsilon}(s)\right\|_{L^{1}(\mathbb{R})} \leq T V\left(u_{0}\right)\left(\left\|f^{\prime}\right\|_{\infty}+\varepsilon\right)|t-s| .
$$

As follows from a standard diagonal procedure and from Helly's theorem, we get the existence of some measurable function $u$ in $L^{\infty}\left(\mathbb{R}^{+} ; B V(\mathbb{R})\right) \cap \mathcal{L} i p\left(\mathbb{R}^{+} ; L^{1}(\mathbb{R})\right)$, such that for some sequence $\left(u_{\varepsilon_{\nu}}\right)_{\nu \in \mathbb{N}}$, with $\varepsilon_{\nu} \rightarrow 0^{+}$as $\nu \rightarrow+\infty$, one has the following convergences:

$$
\text { for all } t \in \mathbb{R}^{+}, \quad u_{\varepsilon_{\nu}}(t, \cdot) \rightarrow u(t, \cdot) \text { in } L_{l o c}^{1}(\mathbb{R}),
$$

and

$$
u_{\varepsilon_{\nu}} \rightarrow u \text { in } L_{l o c}^{1}\left(\mathbb{R}^{+} \times \mathbb{R}\right) .
$$

It remains only to prove that $u$ is an entropy solution. Recalling (25) and taking the additional displacement of the algorithm into account, we deduce that any front (traveling at speed $\sigma$ ) has approximately the correct speed in the sense that:

$$
|\lambda-\sigma| \leq 3 \varepsilon, \quad \forall \lambda \in\left[\bar{\lambda}^{\min }\left(u_{l} ; u_{r}\right), \bar{\lambda}^{\max }\left(u_{l} ; u_{r}\right)\right]
$$

Deducing that $u$ is an entropy solution from (32) is entirely similar to [8, Lemma 5.6]; for the sake of completeness, we briefly sketch the proof. It is sufficient to prove that for any convex entropy couple $(\eta, q)$, for any $\varphi \in$ $C_{0}^{\infty}\left(\mathbb{R}^{+} \times \mathbb{R}\right)$ with $\varphi \geq 0$, the following inequality holds:

$$
\iint_{\mathbb{R}^{+*} \times \mathbb{R}}\left[\eta\left(u_{\varepsilon}\right) \varphi_{t}+q\left(u_{\varepsilon}\right) \varphi_{x}\right] d t d x=\int_{0}^{T} \sum_{\alpha}\left(\dot{x}_{\alpha}(t)\left[\eta\left(u^{\varepsilon}\right)\right]_{\alpha}(t)-\left[q\left(u^{\varepsilon}\right)\right]_{\alpha}(t)\right) \varphi\left(t, x_{\alpha}(t)\right) d t \geq-\mathcal{O}(\varepsilon),
$$

where the sum is over all fronts at time $t$, each front $\alpha$ is described by $t \mapsto x_{\alpha}(t)$ during its time interval of existence $\left[T_{1}^{\alpha}, T_{2}^{\alpha}\right]$, and the brackets designate the jump of a quantity across $\alpha$. We introduce $U^{\alpha}(t, x)$ on $\left[T_{1}^{\alpha}, T_{2}^{\alpha}\right] \times \mathbb{R}$ equal to $u_{\varepsilon}\left(\alpha^{-}\right)$for $x<x_{\alpha}(t)$ and to $u_{\varepsilon}\left(\alpha^{+}\right)$for $x>x_{\alpha}(t)$. Now it is sufficient to prove that the following inequality holds for each front $\alpha$ :

$$
\begin{aligned}
\int_{T_{1}^{\alpha}}^{T_{2}^{\alpha}}\left(\dot{x}_{\alpha}(t)\left[\eta\left(u_{\varepsilon}\right)\right]_{\alpha}(t)\right. & \left.-\left[q\left(u_{\varepsilon}\right)\right]_{\alpha}(t)\right) \varphi\left(t, x_{\alpha}\right) d t \\
& =\int_{T_{1}^{\alpha}}^{T_{2}^{\alpha}} \int_{\mathbb{R}}\left(\eta\left(U^{\alpha}\right) \varphi_{t}+q\left(U^{\alpha}\right) \varphi_{x}\right) d t d x-\left[\int_{\mathbb{R}} \eta\left(U^{\alpha}\right) \varphi(\cdot, x) d x\right]_{T_{1}^{\alpha}}^{T_{2}^{\alpha}} \\
& \gtrsim-\varepsilon\left|u_{\varepsilon}\left(\alpha^{+}\right)-u_{\varepsilon}\left(\alpha^{-}\right)\left\|T_{2}^{\alpha}-T_{1}^{\alpha} \mid\right\| \varphi \|_{C^{1}} .\right.
\end{aligned}
$$

(Once (34) is established, it remains to sum according to $\alpha$, and to let $\varepsilon \rightarrow 0^{+}$.)

Now to get (34), introduce the actual solution $\widehat{U}^{\alpha}$ of the Riemann problem $\left(u\left(\alpha^{-}\right), u\left(\alpha^{+}\right)\right)$, centered at the point $\left(T_{1}^{\alpha}, x_{\alpha}\left(T_{1}^{\alpha}\right)\right)$ and estimate the integrals in (33). When we replace $U^{\alpha}$ with $\widehat{U}^{\alpha}$ in (33) we obtain a nonnegative result by the admissibility of the actual solution $\widehat{U}^{\alpha}$; it remains to estimate the errors. For the first (resp. second) integral, the integrand generates an error of size $\mathcal{O}(1)\left|u_{\varepsilon}\left(\alpha^{+}\right)-u_{\varepsilon}\left(\alpha^{-}\right)\right|\|\varphi\|_{C^{1}}$, and thanks to (32) we see that this difference lies in a triangle of area $\mathcal{O}(1) \varepsilon\left|T_{2}-T_{1}\right|$ (resp. in an interval of length $\left.\mathcal{O}(1) \varepsilon\left|T_{2}-T_{1}\right|\right)$, which concludes the proof.

\section{Preliminary material}

Before getting to the proof of Proposition 1, let us introduce some tools which we will use during the proof.

\section{$3.1 \quad$ Terminology}

Now that we have defined the front-tracking approximations, let us add some terminology. We will call a positive (resp. a negative) front, a front with left and right states $\left(u_{-}, u_{+}\right)$such that $u_{-}\left\langle u_{+}\left(\right.\right.$resp. $\left.\left.u_{-}\right\rangle u_{+}\right)$. Note that in general this distinction is not very relevant when the flux is general (it is essentially a matter of parameterization). On the other hand, in the case of a convex flux, positive fronts make the left-hand side of Oleinik's inequality positive while negative fronts make it negative. Hence, it is natural here to distinguish positive and negative fronts, because of the particular form of the right-hand side of (11): roughly speaking, in 
the situation where the additional error term in (11) vanishes, positive fronts are rarefaction fronts (which are important) while negative fronts are shocks (which should be ignored).

At an interaction point $P \in \mathbb{R}^{+*} \times \mathbb{R}$, we will call the total variation dissipated at $P$, and denote $[\Delta T V](P)$ or even $\Delta T V$ when there is no ambiguity, the following value:

$$
[\Delta T V](P):=\left|u_{r}^{P}-u_{m}^{P}\right|+\left|u_{m}^{P}-u_{l}^{P}\right|-\left|u_{r}^{P}-u_{l}^{P}\right| \quad(>0) .
$$

Finally, additionally to the inner speed variation of a front $\left(u_{-}, u_{+}\right)$, we will define the outer speed variation (osv in short) of a front as the following value:

$$
\tau\left(u_{-}, u_{+}\right):=f^{\prime}\left(u_{+}\right)-f^{\prime}\left(u_{-}\right)
$$

Note that, unlike the inner speed variation, the outer speed variation can likely be negative. Also remark that Lax's inequalities

involve the relation

$$
f^{\prime}\left(u_{-}\right) \geq \bar{\lambda}^{\min }\left(u_{-}, u_{+}\right) \text {and } f^{\prime}\left(u_{+}\right) \leq \bar{\lambda}^{\max }\left(u_{-}, u_{+}\right)
$$

$$
\tau\left(u_{-}, u_{+}\right) \leq \vartheta\left(u_{-}, u_{+}\right)
$$

\subsection{Interaction estimates on the speeds}

In this paragraph, we state some preliminary lemmas, which compare the speeds of the incoming and outgoing fronts (not their inner speed variation as in Proposition 4) across an interaction point. The first two lemmas are independent of the previous approximating scheme; the last one is valid for an $\varepsilon$ front-tracking approximation constructed by the above process. Of course, one cannot expect the difference of speeds across an interaction to be measured by an interaction potential term; the interaction estimates which we present here are inequalities, with various "error terms".

Lemma 2 (Monotone interactions). Consider $u_{l}<u_{m}<u_{r}$ (or $\left.u_{l}>u_{m}>u_{r}\right)$. Then:

$$
\begin{gathered}
\bar{\lambda}^{\min }\left(u_{l} ; u_{r}\right) \leq \bar{\lambda}^{\min }\left(u_{l} ; u_{m}\right), \\
\bar{\lambda}^{\max }\left(u_{m} ; u_{r}\right) \leq \bar{\lambda}^{\max }\left(u_{l} ; u_{r}\right) .
\end{gathered}
$$

Moreover, we have the two following (compatible) possibilities:

$$
\bar{\lambda}^{\mathrm{min}}\left(u_{l} ; u_{r}\right)=\bar{\lambda}^{\mathrm{min}}\left(u_{l} ; u_{m}\right) \text { or } \bar{\lambda}^{\mathrm{min}}\left(u_{m} ; u_{r}\right) \leq \bar{\lambda}^{\mathrm{min}}\left(u_{l} ; u_{r}\right),
$$

and

$$
\bar{\lambda}^{\max }\left(u_{m} ; u_{r}\right)=\bar{\lambda}^{\max }\left(u_{l} ; u_{r}\right) \text { or } \bar{\lambda}^{\max }\left(u_{l} ; u_{r}\right) \leq \bar{\lambda}^{\max }\left(u_{l} ; u_{m}\right)
$$

Lemma 3 (Non-monotone interactions, positive outgoing fronts). Consider three states $u_{l}, u_{m}$ and $u_{r}$, with either $u_{l}<u_{r}<u_{m}$ or $u_{m}<u_{l}<u_{r}$. Consider the larger incoming front $\left(u_{-} ; u_{+}\right)$, that is $\left(u_{-} ; u_{+}\right):=\left(u_{l} ; u_{m}\right)$ in the former case, $\left(u_{-} ; u_{+}\right):=\left(u_{m} ; u_{r}\right)$ in the latter. Then the following holds:

$$
\begin{aligned}
& \bar{\lambda}^{\min }\left(u_{l} ; u_{r}\right) \geq \bar{\lambda}^{\min }\left(u_{-} ; u_{+}\right)-\left(\left\|\left(f^{\prime \prime}\right)_{-}\right\|_{\infty} / 2\right) \Delta T V, \\
& \bar{\lambda}^{\max }\left(u_{l} ; u_{r}\right) \leq \bar{\lambda}^{\max }\left(u_{-} ; u_{+}\right)+\left(\left\|\left(f^{\prime \prime}\right)_{-}\right\|_{\infty} / 2\right) \Delta T V .
\end{aligned}
$$

Lemma 4 (Non-monotone interactions, negative outgoing fronts). Consider, in an $\varepsilon$ front-tracking approximation constructed by the above algorithm, two fronts $\left(u_{l}, u_{m}\right)$ and $\left(u_{m} ; u_{r}\right)$ which are interacting, with $u_{m}<u_{r}<u_{l}$ or $u_{r}<u_{l}<u_{m}$. Then the following holds:

- Case 1: $u_{m}<u_{r}<u_{l}$. In that case, call $\left(\tilde{u}, u_{r}\right)$ the rightmost outgoing front. One has:

$$
\begin{aligned}
& \bar{\lambda}^{\min }\left(u_{l} ; u_{r}\right) \geq \bar{\lambda}^{\min }\left(u_{m} ; u_{r}\right), \\
& \bar{\lambda}^{\max }\left(u_{l} ; u_{r}\right) \leq \bar{\lambda}^{\max }\left(u_{m} ; u_{r}\right)+\left[f^{\prime}(\tilde{u})-f^{\prime}\left(u_{r}\right)\right]_{+}+2 \varepsilon .
\end{aligned}
$$

- Case 2: $u_{r}<u_{l}<u_{m}$. In that case, call $\left(u_{l}, \tilde{u}\right)$ the leftmost outgoing front. One has:

$$
\begin{aligned}
& \bar{\lambda}^{\min }\left(u_{l} ; u_{r}\right) \geq \bar{\lambda}^{\min }\left(u_{l} ; u_{m}\right)-\left[f^{\prime}\left(u_{l}\right)-f^{\prime}(\tilde{u})\right]_{+}-2 \varepsilon, \\
& \bar{\lambda}^{\max }\left(u_{l} ; u_{r}\right) \leq \bar{\lambda}^{\max }\left(u_{l} ; u_{m}\right)+2 \varepsilon .
\end{aligned}
$$

Remark 4. The asymmetry between Lemmas 3 and 4 comes from the fact that we want to have an error term relying on $\left\|\left(f^{\prime \prime}\right)-\right\|_{\infty}$. Clearly, one has an equivalent statement of Lemma 3 for negative outgoing fronts, when $\left\|\left(f^{\prime \prime}\right)_{+}\right\|_{\infty}$ replaces $\left\|\left(f^{\prime \prime}\right)_{-}\right\|_{\infty}$. Note however that one cannot expect estimates such as (41)-(42) to be true in the context of negative outgoing fronts (even if we replace $\left\|\left(f^{\prime \prime}\right)_{-}\right\|_{\infty}$ by any other factor vanishing with $\left.\left(f^{\prime \prime}\right)_{-}\right)$: this is easily seen by considering a convex flux.

We postpone the proofs of Lemmas 2, 3 and 4 to Section 5. 


\subsection{Origin of the surplus of inner speed variation}

In this paragraph, we discuss how a front can have acquired an inner speed variation larger than $\varepsilon$ (which as explained in Section 2, one can consider as a "surplus"). Consider a front $\alpha$ at time $t>0$. To such a front, we associate an inner speed variation source tree, which we will denote by $\mathcal{T}(\alpha)$, according to the following algorithm:

- we start from the "root" front $\alpha$ : if $\vartheta_{\alpha} \leq \varepsilon$ we stop immediately the tree, that is we take $\mathcal{T}(\alpha):=\{\alpha\}$; else we go backwards in time, and:

- at each interaction point that we meet:

- if it is a point of monotone interaction (hence using the non-splitting strategy), we add as branches to the tree all the incoming fronts that have an inner speed variation larger than $\varepsilon$,

- if it is a non-splitting point of non-monotone interaction, we add as a branch to the tree the incoming front that has the same sign as the outgoing front, if it has an inner speed variation larger than $\varepsilon$; else, the branch stops here,

- if this point is a splitting point, we use the convention that the branch stops here. It is not difficult to observe that in fact, this last case cannot happen, because we only follow fronts with an inner speed variation larger than $\varepsilon$.

By convention we consider the tree as a set of fronts (which are connected one to another); hence we consider that two trees intersect when they have a front in common (not just an interaction point). Note that according to the above algorithm, all branches stop before $t=0$ since all fronts outgoing from time $t=0$ have an inner speed variation less than or equal to $\varepsilon$. Also note that the only case where a front having an inner speed variation less than or equal to $\varepsilon$ belongs to such a tree is when it is equal to the whole tree. Finally, remark that in such a tree, all fronts share the same sign. We give an example of how such a tree could look like in Figure 1.

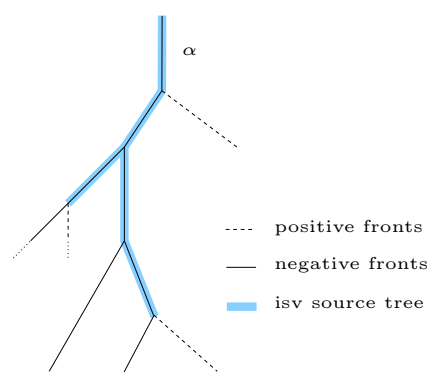

Figure 1: An example of inner speed variation source tree

Denote $\mathcal{V}(\alpha)$ the set of vertices of $\mathcal{T}(\alpha)$, where the top of the tree (that is, the interaction point to which the root $\alpha$ arrives) is excluded, but the vertices at the bottom of the leaves are included. We claim the following.

Lemma 5. 1. The construction is transitive in the sense that

$$
\beta \in \mathcal{T}(\alpha) \text { and } \gamma \in \mathcal{T}(\beta) \Rightarrow \gamma \in \mathcal{T}(\alpha)
$$

2. The inner speed variation source trees are disjoints in the sense that, given two fronts $\alpha$ and $\beta$, one has

$$
\mathcal{T}(\alpha) \cap \mathcal{T}(\beta) \neq \emptyset \Rightarrow \mathcal{T}(\alpha) \subset \mathcal{T}(\beta) \text { or } \mathcal{T}(\beta) \subset \mathcal{T}(\alpha) .
$$

3. Given a front $\alpha$, one can estimate the inner speed variation of $\alpha$ by

$$
\vartheta_{\alpha}-\varepsilon \leq \sqrt{\left\|\left(f^{\prime \prime}\right)-\right\|_{\infty}\left\|f^{\prime \prime}\right\|_{\infty}} \sum_{P \in \mathcal{V}(\alpha)}[\Delta T V](P) .
$$

The proof of Lemma 5 is postponed to Section 5 . 


\subsection{A scenario for the origin of outer speed variation}

In this paragraph, we describe a sort of "tree" which is intended to play the same role for the outer speed variation as the tree described in Paragraph 3.3 does for the inner speed variation. However, it will play this role only in a particular situation, as we will see.

Given a negative front $\alpha$ at time $t>0$ with a positive outer speed variation, we draw its outer speed variation source tree, which we denote by $\widehat{\mathcal{T}}(\alpha)$, as follows. (By convention, if $\tau(\alpha) \leq 0$ or the front is positive, we stop immediately and fix $\widehat{\mathcal{T}}(\alpha):=\{\alpha\}$.) Else, starting from the root $\alpha$, we go backward in time, and at each interaction point:

- if the interaction point is a monotone interaction point, add to the tree the incoming fronts that have a positive outer speed variation;

- if the interaction point is a non-monotone interaction point, stop the branch here.

- stop at $t=0$ of course.

Remark 5. Since fronts outgoing from a splitting (non-monotone) interaction point can likely be selected in the above process, two selected branches can in fact rejoin at such a point. See for instance Figure 2. Consequently this selection of fronts should be more accurately called a graph. However we see from the above algorithm that the tree stops at a point of this type. Hence it suffices to consider that we artificially disconnect all fronts outgoing from a splitting point to perceive this graph as a tree.

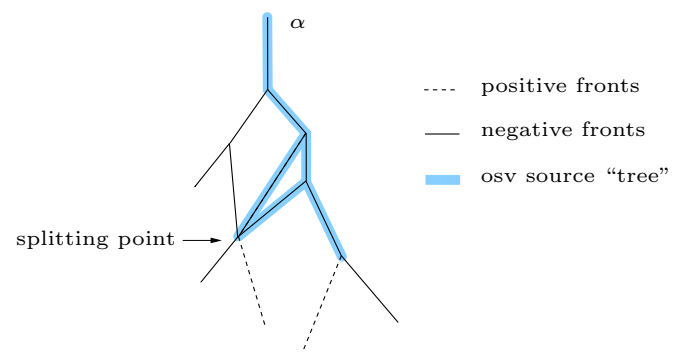

Figure 2: An example of outer speed variation source tree

Remark that in this tree, again, all fronts share the same sign. Denote $\widehat{\mathcal{L}}(\alpha)$ the set of the leaves of $\widehat{\mathcal{T}}(\alpha)$, that is, the fronts at which the tree stops (the root $\alpha$ being of course excluded). We call such a leaf good if and only if it is generated at a positive time and one of the following possibilities occurs:

- it emerges from a non monotone interaction point $u_{m}<u_{r}<u_{l}$ and the front considered is not the rightmost one,

- it emerges from a non monotone interaction point $u_{r}<u_{l}<u_{m}$ and the front considered is not the leftmost one.

(In particular, a leaf cannot be good if it emerges from a non monotone interaction point using the no-splitting strategy.) Call a good tree a non-trivial tree whose all leaves are good. Call inner vertices the vertices of the tree, except the top of the tree and the bottom of its leaves. It follows from the previous construction that all inner vertices of a good tree are monotone interaction points, and hence use the no splitting strategy.

We claim the following.

Lemma 6. 1. The construction is transitive:

$$
\beta \in \widehat{\mathcal{T}}(\alpha) \text { and } \gamma \in \widehat{\mathcal{T}}(\beta) \Rightarrow \gamma \in \widehat{\mathcal{T}}(\alpha)
$$

2. The outer speed variation source trees are disjoints in the sense that, given two fronts $\alpha$ and $\beta$, one has

$$
\widehat{\mathcal{T}}(\alpha) \cap \widehat{\mathcal{T}}(\beta) \neq \emptyset \Rightarrow \widehat{\mathcal{T}}(\alpha) \subset \widehat{\mathcal{T}}(\beta) \text { or } \widehat{\mathcal{T}}(\beta) \subset \widehat{\mathcal{T}}(\alpha) .
$$

3. Let $\alpha$ be a negative front. Assume that the tree $\widehat{\mathcal{T}}(\alpha)$ is good. Then

$$
\tau(\alpha) \leq \sum_{\beta \in \widehat{\mathcal{L}}(\alpha)} \vartheta(\beta) .
$$

We prove Lemma 6 in Section 5 . 


\section{Proof of Proposition 1}

\subsection{Starting point}

We consider $u_{0} \in L^{1}(\mathbb{R}) \cap B V(\mathbb{R})$, and a sequence $\left(u_{\varepsilon_{\nu}}\right)$ of $\varepsilon_{\nu}$-front-tracking approximations converging to the entropy solution $u$ of (1)-(2), in the sense of (30)-(31).

We consider $T>0$ and we will first establish the following inequality on $u_{\varepsilon_{\nu}}$ :

$$
\begin{aligned}
& f^{\prime}\left(u_{\varepsilon_{\nu}}(T, y)\right)-f^{\prime}\left(u_{\varepsilon_{\nu}}(T, x)\right) \leq \frac{y-x}{T}+\sqrt{\left\|\left(f^{\prime \prime}\right)-\right\|_{\infty}\left\|f^{\prime \prime}\right\|_{\infty}}\left[T V\left(u_{\varepsilon_{\nu}}(0, \cdot)\right)-T V\left(u_{\varepsilon_{\nu}}(T, \cdot)\right)\right] \\
&+\mathcal{O}\left(\varepsilon_{\nu}\right) \text { for } x \leq y .
\end{aligned}
$$

Then, we will pass to the limit and extend the result to slightly more general $u_{0}$.

To establish (49), we consider $\nu \in \mathbb{N}^{*}, x<y$ and $T>0$. Now, consider $u_{\varepsilon_{\nu}}$ at time $T$ : if there is no front in the interval $\{T\} \times(x, y]$, there is nothing to prove since $f^{\prime}\left(u_{\varepsilon_{\nu}}(T, y)\right)=f^{\prime}\left(u_{\varepsilon_{\nu}}(T, x)\right)$. If there is only one front between $x$ and $y$, the result is true with only $\mathcal{O}\left(\varepsilon_{\nu}\right)$ on the right hand side. This follows directly from $(25)$ and (36).

Henceforth, we suppose that there are at least two fronts inside $(x, y]$. It is clear that to establish (49), it is sufficient to establish it when $x$ and $y$ are located exactly on separated fronts, and the values to be considered in the left hand side of (49) are $f^{\prime}\left(u_{\varepsilon_{\nu}}\left(T, x^{-}\right)\right)$and $f^{\prime}\left(u_{\varepsilon_{\nu}}\left(T, y^{+}\right)\right)$. (If necessary, move $x$ to the right and $y$ to the left: this does not modify the left hand side, and reduces the right hand side.) From now on, we suppose that $x$ and $y$ are located on different fronts, and aim at proving (49) with this convention. To simplify the notations, we will drop the index $\varepsilon_{\nu}$ from $u_{\varepsilon_{\nu}}$ until Paragraph 4.4.4; when $\varepsilon_{\nu}$ itself is concerned, we will drop the index $\nu$ from it. Also, unless otherwise stated, the values of the approximations $u_{\varepsilon_{\nu}}(t, \cdot)$ at the point $x$ will be the ones from the left.

\subsection{Tracing fronts backwards}

In this paragraph, we explain how we choose to "trace", starting from $x$ and $y$, two continuous lines $\gamma^{x}$ and $\gamma^{y}$, made of fronts, backwards in time. This will crucially depend on the sign (positive or negative) of the front that we consider. Recall that the difference of treatment of positive and negative fronts can be explained by the fact that we want an error of the form $\sqrt{\left\|\left(f^{\prime \prime}\right)_{-}\right\|_{\infty}\left\|f^{\prime \prime}\right\|_{\infty}} \Delta T V$. In particular, negative fronts which do not intervene in Oleinik's inequality in the convex case, should more or less be considered as "error generators" here.

The tracing algorithm is carried out in several steps:

- for positive fronts, the algorithm selects a unique front path,

- for negative fronts, the algorithm selects a front tree (which is not the tree considered in Paragraph 3.3, and not quite the one of Paragraph 3.4),

- then another step (which we call "postprocessing") is performed on each tree selected above for negative fronts (if any): either it singles out a front path inside this tree, or it tells to abandon this tree and repeat the above steps on the "next" front living at time $T$.

\subsubsection{Tracing positive fronts backwards.}

Now, supposing that the front located at $x$ or $y$ is positive, we choose to "extend" it as a path for times $t \leq T$ according to the following rule: follow backwards in time the front which is located at $x$ (resp. $y$ ), and at each interaction point:

- if the interaction point is a non-monotone interaction point, follow the incoming front that is positive;

- if the interaction point is a monotone interaction point:

- for the front path coming from $x$, pick the slowest (i.e. the right) incoming front,

- for the front path coming from $y$, pick the fastest incoming front in the following particular sense: choose the one which maximizes $\bar{\lambda}^{\max }\left(u_{-}, u_{+}\right)$. (Choose arbitrarily in case of equality.)

Call $\gamma^{x}$ (resp. $\gamma^{y}$ ) the resulting piecewise affine path. Apply the above rule until $\gamma^{x}$ (resp. $\gamma^{y}$ ) reaches $t=0$. (It may happen that $\gamma^{x} \cap \gamma^{y} \neq \emptyset$.) In the case under view, we fix $\bar{\gamma}^{x}:=x$ (resp. $\bar{\gamma}^{y}:=y$ ).

Remark 6. Due to the convention that all fronts travel at the minimum speed of the wave packet, we cannot affirm that the incoming front which maximizes $\bar{\lambda}^{\max }\left(u_{-}, u_{+}\right)$is the left one. One can imagine situations where this is not the case; this is due to the approximation, and is likely to disappear as $\varepsilon \rightarrow 0^{+}$. 


\subsubsection{Tracing negative fronts backwards.}

Now, we suppose that the front located at $x$ or $y$ is negative. We choose to extend it for times $t \leq T$ as a tree. This tree is an extension of the tree discussed in Paragraph 3.4; we will call it extended outer speed variation source tree. This is done according to the following rules: if the front has a nonpositive outer speed variation, stop the tree immediately; else follow backwards the front located at $x$ (resp. $y$ ), and at each interaction:

- if the interaction point is a monotone interaction point, add to the tree the incoming fronts that have a positive outer speed variation;

- if the interaction point is a non-monotone interaction point:

- if it is a non-splitting interaction point, add the positive incoming front, and extend this positive front to $t=0$ with the rule of Paragraph 4.2.1,

- if it is a splitting interaction point, then

* if $u_{m}<u_{r}<u_{l}$ and the front which we want to extend is the rightmost one, add the positive incoming front, and extend this positive front with the above rule for positive fronts,

* if $u_{r}<u_{l}<u_{m}$ and the front which we want to extend is the leftmost one, add the positive incoming front, and extend this positive front with the above rule for positive fronts,

* in all the other cases, stop the branch of the tree here.

Of course, all branches that we select must also stop when they touch $t=0$.

Remark 7. Let us remark that the extended osv source tree coincides with the osv source tree if and only if we do not add positive fronts, that is, if and only if all the leaves of the osv source tree which do not touch $t=0$ are good. Hence an extended osv source tree either touches $t=0$ or coincides with a "good" osv source tree.

\subsubsection{Postprocessing.}

Now in a second step, we simplify the tree(s) generated by a negative front according to the following rules. At the beginning, we fix $\bar{\gamma}^{x}:=x$ and $\bar{\gamma}^{y}:=y$.

- If the tree contains no branch that reaches $t=0$ or if it corresponds to a single front with non positive outer speed variation, "abandon" it, that is, replace $\bar{\gamma}^{x}$ with the next front on the right of $\bar{\gamma}^{x}$ at $t=T$ (if we considered the $x$-tree) or on the left of $\bar{\gamma}^{y}$ (if we considered the $y$-tree), and iterate the process. Naturally, stop if the $x$-front and the $y$-front considered at time $T$ are the same, i.e. if $\bar{\gamma}^{x}=\bar{\gamma}^{y}$.

- If on the contrary, we are in a situation where the tree under view reaches $t=0$, then we select a front path inside it according to the following rules. Start again from the front at time $T$ (located at $\bar{\gamma}^{x}$ and $\bar{\gamma}^{y}$, respectively), and go backwards in time in the tree. It follows from the algorithm of Paragraph 4.2.2 that in this process, we will only meet monotone interaction points, unless the branch becomes a positive front path. At each interaction point:

- if one and only one of the incoming fronts belongs to the tree, of course we follow this one, (this includes in particular non-monotone interaction points, and positive parts of the tree),

- if the two incoming fronts belong to the tree: we consider the subtrees generated respectively by these two incoming fronts:

$*$ if only one of the subtrees still satisfies the condition that it reaches $t=0$, follow this branch,

$*$ if both subtrees satisfy the condition that they reach $t=0$, then what we do depends on the side (left or right) of the tree that we consider:

- for a $x$-tree, pick the left incoming front,

- for a $y$-tree, pick the right incoming front.

At the end of the process, either $\bar{\gamma}^{x}=\bar{\gamma}^{y}$, (i.e. we have abandoned all the fronts between $x$ and $y$ except the last one corresponding to $\bar{\gamma}^{x}=\bar{\gamma}^{y}$ ) or we get two distinct front paths $\gamma^{x}$ and $\gamma^{y}$ that both meet $t=0$ and which start respectively from $\bar{\gamma}^{x}$ and $\bar{\gamma}^{y}$ (see for instance Figure 6 in Paragraph 4.4 below for an example of the latter case). Call $\mathcal{A}$ the set of all fronts living at time $T$, whose tree has been abandoned in the process; we will call such fronts abandoned as well. 


\subsubsection{Structure of the proof.}

Now the rest of proof is done in two steps:

- First, we establish (49) in the case where we have singled out two front paths $\gamma^{x}$ and $\gamma^{y}$ that both meet the axis $t=0$ (possibly meeting each other before touching $t=0$ ). In other words, we estimate in this case

$$
f^{\prime}\left(\left(\bar{\gamma}^{y}\right)^{+}\right)-f^{\prime}\left(\left(\bar{\gamma}^{x}\right)^{-}\right)=f^{\prime}\left(\gamma^{y}(T)^{+}\right)-f^{\prime}\left(\gamma^{x}(T)^{-}\right) .
$$

While doing this, we actually do not take all the dissipation of total variation into account.

- Next, we prove that the trees that we "abandoned" in the above process can be taken into account in the rest of the total variation dissipation (this will include the case when $\bar{\gamma}^{x}=\bar{\gamma}^{y}$ ). In other words, we estimate

$$
f^{\prime}(y)-f^{\prime}(x)-\left[f^{\prime}\left(\gamma^{y}(T)^{+}\right)-f^{\prime}\left(\gamma^{x}(T)^{-}\right)\right],
$$

with the rest of the total variation dissipation.

The largest part is done in the following paragraph, where the central proposition is stated and proved.

\subsection{The central proposition}

\subsubsection{Statement}

In this paragraph and until Paragraph 4.4.3, we consider that the previous process yielded two distinct fronts $\bar{\gamma}^{x}$ and $\bar{\gamma}^{y}$ and drew from them two front paths $\gamma^{x}$ and $\gamma^{y}\left(\right.$ with $\left.\bar{\gamma}^{x}=\gamma^{x}(T)<\gamma^{y}(T)=\bar{\gamma}^{y}\right)$.

Let us call $\dot{\gamma}^{x}(t)$ (resp. $\dot{\gamma}^{y}(t)$ ) the speed of the front path $\gamma^{x}$ (resp. $\gamma^{y}$ ) at time $t \in[0, T]$. This is uniquely defined except at interaction times. If $\gamma^{x}$ and $\gamma^{y}$ meet at a positive time, we call $\tilde{t}$ their meeting time, that is, the largest time $t$ for which $\gamma^{x}(t)=\gamma^{y}(t)$. In other words, when we follow $\gamma^{x}$ and $\gamma^{y}$ backwards in time starting from $t=T$, this is the first time the two front paths meet. Else, we define $\tilde{t}:=0$. It is a consequence of the convention of Paragraph 4.2.2 that $\tilde{t}=0$ except possibly if both $\gamma^{x}$ and $\gamma^{y}$ represent positive fronts at the time they meet (at a splitting interaction point with negative outgoing fronts, only one of the outgoing fronts is extended backwards by the above process, and is a candidate to be $\gamma^{x}$ or $\gamma^{y}$ ).

In the sequel, it will be convenient to express that only a part of the total variation dissipation at an interaction point $P$ is used in an estimate, the "rest" of the total dissipation at the interaction point being possibly used to estimate another term. For this purpose, we introduce $\mathcal{I}(T)$ as the set of all interaction points between times 0 and $T$. Given an application $\mathcal{N}: \mathcal{I}(T) \rightarrow \mathbb{N}^{*}$, we introduce $\mathfrak{I}_{\mathcal{N}}(T)$ as the set of all interaction points between times 0 and $T$, where each interaction point $P$ is counted $\mathcal{N}(P)$ times:

$$
\mathfrak{I}_{\mathcal{N}}(T):=\bigcup_{P \in \mathcal{I}(T)} \bigcup_{i=1}^{\mathcal{N}(P)}\{(P, i)\} .
$$

Call the second coordinate of $(P, i)$ a marker, and $(P, i)$ a representative of $P$.

We will also introduce an allocation of the total variation dissipation, that is, for each $(P, i) \in \mathfrak{I}_{\mathcal{N}}(T)$, we will determine the $i$-th elementary part of total variation dissipation at $P$ (which we will denote by $\Delta T V(P, i)$ ) in such a way that

$$
\Delta T V(P, i) \geq 0 \text { and } \sum_{i=1}^{\mathcal{N}(P)} \Delta T V(P, i)=\Delta T V(P) .
$$

This allocation is obvious when $\mathcal{N}(P)=1$. Finally, since there is no total variation dissipation at monotone interaction points, the function $\mathcal{N}$ can be defined arbitrarily at such points; this has no importance.

The main result of Paragraph 4.3 is the following.

Proposition 5. Given $T>0$, there exist an application $\mathcal{N}: \mathcal{I}(T) \rightarrow \mathbb{N}^{*}$ and an allocation of $\Delta T V$, such that for $\gamma^{x}$ and $\gamma^{y}$ constructed as above, there exist subsets $\mathcal{D}(\alpha)$ of $\mathfrak{I}_{\mathcal{N}}(T)$ defined for all front $\alpha$ living at time $T$, such that the following properties hold. One has the following estimates on the speeds of the fronts: for all $t \in[\tilde{t}, T]$ :

$$
\begin{aligned}
& \dot{\gamma}^{x}(t) \leq \dot{\gamma}^{x}(T)+\sqrt{\left\|\left(f^{\prime \prime}\right)-\right\|_{\infty}\left\|f^{\prime \prime}\right\|_{\infty}} \sum_{(P, i) \in \mathcal{D}\left(\bar{\gamma}^{x}\right)} \Delta T V(P, i)+8 \varepsilon, \\
& \dot{\gamma}^{y}(t) \geq \dot{\gamma}^{y}(T)-\sqrt{\left\|\left(f^{\prime \prime}\right)-\right\|_{\infty}\left\|f^{\prime \prime}\right\|_{\infty}} \sum_{(P, i) \in \mathcal{D}\left(\bar{\gamma}^{y}\right)} \Delta T V(P, i)-8 \varepsilon .
\end{aligned}
$$

One has the following estimates on the osv of abandoned fronts:

$$
\forall \alpha \in \mathcal{A}, \quad \tau(\alpha) \leq \sqrt{\left\|\left(f^{\prime \prime}\right)_{-}\right\|_{\infty}\left\|f^{\prime \prime}\right\|_{\infty}} \sum_{(P, i) \in \mathcal{D}(\alpha)} \Delta T V(P, i) .
$$

Finally, the subsets $\mathcal{D}$ satisfy:

if $\alpha$ and $\beta$ are two distinct fronts living at time $T$, then $\mathcal{D}(\alpha) \cap \mathcal{D}(\beta)=\emptyset$. 
The rest of Section 4.3 is devoted to the proof of Proposition 5. This proof is split as follows. First, we describe the function $\mathcal{N}$ and the allocation of total variation dissipation. Next, we will explain why one has estimates of the type (51)-(52) - the sets $\mathcal{D}\left(\bar{\gamma}^{x}\right), \mathcal{D}\left(\bar{\gamma}^{y}\right)$ are selected during the process - ; there are several cases to consider, according to the sign of the front path and to its side (left for $\gamma^{x}$ or right for $\gamma^{y}$ ). Then, we will select $\mathcal{D}(\alpha)$ for abandoned fronts $\alpha \in \mathcal{A}$ and justify (53). Finally we prove the central condition (54) on the sets $\mathcal{D}$ selected before. We write these parts in different paragraphs.

\subsubsection{The function $\mathcal{N}$ and the allocation of total variation dissipation.}

For a splitting point of interaction $Q$, we define $\nu_{Q}$ as the number of fronts outgoing from $Q$. We will generically call $\mu_{1}, \ldots, \mu_{\nu_{Q}}$, these fronts, numbered from left to right; when $\beta$ is such an outgoing front, we note $\iota_{\beta} \in$ $\left\{1, \ldots, \nu_{Q}\right\}$ the index such that $\beta=\mu_{\iota_{\beta}}$.

Let us first describe the function $\mathcal{N}$ that we will use. We consider the set $\mathcal{S}$ of all splitting interaction points $Q$, whose outgoing fronts are negative.

- For any $Q \in \mathcal{S}$, we consider the isv source tree of the larger incoming front at $Q$ (that is to say, the negative one); on the vertices of this tree and on $Q$, we set $\mathcal{N}=\nu_{Q}-1$.

We claim that at most one value of $\mathcal{N}$ is given to each interaction point $R$ by this convention. Suppose indeed that two different values are given to a point $R$.

- If $R \in \mathcal{S}$, then $\mathcal{N}(R)=\nu_{R}-1$, and no other value can be given to it, since no splitting point can be the vertex of an isv source tree (only possibly the top of it).

- Suppose now $R \notin \mathcal{S}$. Hence there are $Q_{1}$ and $Q_{2}$ in $\mathcal{S}$ which have this point inside the isv source tree of their negative incoming front. Since $R \notin \mathcal{S}$ these two trees have a front in common. By Lemma 5 , one of these isv source trees is included in the other one. But this inclusion cannot be strict, because $Q_{1}$ and $Q_{2}$ are splitting points, and there are no splitting point in the inside of an isv source tree.

- Finally, we can choose $\mathcal{N}=1$ everywhere else.

Now concerning the allocation of total variation dissipation: it is trivial for points on which $\mathcal{N}$ equals 1 . On the other points (say, $P$ ), this depends on the nature of the point $Q$ which generated this value of $\mathcal{N}$ (as we just explained, $Q$ is unique). Again we call $\mu_{1}, \ldots, \mu_{\nu_{Q}}$ the fronts outgoing from $Q$, from left to right.

- First case: $u_{r}^{Q}<u_{l}^{Q}<u_{m}^{Q}$. In that case, define

$$
\Delta T V(P, i):=\frac{\vartheta\left(\mu_{i+1}\right)}{\sum_{j=2}^{\nu_{Q}} \vartheta\left(\mu_{j}\right)} \Delta T V(P) \text { for } i=1, \ldots, \nu_{Q}-1 .
$$

- Second case: $u_{m}^{Q}<u_{r}^{Q}<u_{l}^{Q}$. In that case, define

$$
\Delta T V(P, i):=\frac{\vartheta\left(\mu_{i}\right)}{\sum_{j=1}^{\nu_{Q}-1} \vartheta\left(\mu_{j}\right)} \Delta T V(P) \text { for } i=1, \ldots, \nu_{Q}-1 .
$$

The allocation of total variation dissipation described by (55)-(56) includes the case $P=Q$.

\subsubsection{Proof of (51)-(52): positive front paths.}

We consider here a front path $\left(\gamma^{x}\right.$ or $\left.\gamma^{y}\right)$ along which the front is positive during the whole time interval $[\tilde{t}, T]$.

- We begin with the case of the left front path $\gamma^{x}$, and establish (51). Starting from $t=T$, we go backwards in time. To estimate the speed of $\gamma^{x}$, we define

$$
\sigma^{x}(s):=\bar{\lambda}^{\min }\left(\gamma^{x}(s)^{-}, \gamma^{x}(s)^{+}\right) .
$$

It is possible that $\dot{\gamma}^{x}(s) \neq \sigma^{x}(s)$ due to the additional displacement (see the convention in Paragraph 2.2); however we always have, apart from interaction times:

$$
\left|\sigma^{x}(s)-\dot{\gamma^{x}}(s)\right| \leq \varepsilon .
$$

Now, at each time of interaction (say $s$ ) involving $\gamma^{x}$, we have according to the situation: 
- if the interaction located at $\gamma^{x}(s)$ is monotone, then

$$
\sigma^{x}\left(s^{+}\right) \geq \sigma^{x}\left(s^{-}\right),
$$

as follows from Lemma 2 (see in particular (39)) and the choice that we made in Paragraph 4.2.1;

- and if it is non-monotone, then it follows from Lemma 3 that

$$
\sigma^{x}\left(s^{+}\right) \geq \sigma^{x}\left(s^{-}\right)-\frac{\left\|\left(f^{\prime \prime}\right)_{-}\right\|_{\infty}}{2} \Delta T V\left(\gamma^{x}(s)\right) .
$$

(Note that the above inequality is valid whether $P:=\gamma^{x}(s)$ is a splitting point or not, since in all cases

$$
\sigma^{x}\left(s^{+}\right) \geq \bar{\lambda}^{\min }\left(u_{l}^{P}, u_{r}^{P}\right),
$$

that is, the front followed by $\gamma^{x}$ after time $s$ travels at least as fast as the slowest outgoing front.)

Summing over all interactions points inside $\gamma^{x}$ during $(\tilde{t}, T)$, we get (51) in that case, by choosing $\mathcal{D}\left(\bar{\gamma}^{x}\right)$ to be the set of non monotone interaction points on $\gamma^{x}$ during times in $(\tilde{t}, T)$. We remark that $\mathcal{N}$ is equal to 1 on $\mathcal{D}\left(\bar{\gamma}^{x}\right)$ (because points at which $\mathcal{N}$ has values larger than 1 have negative outgoing fronts). We represent the situation in Figure 3.

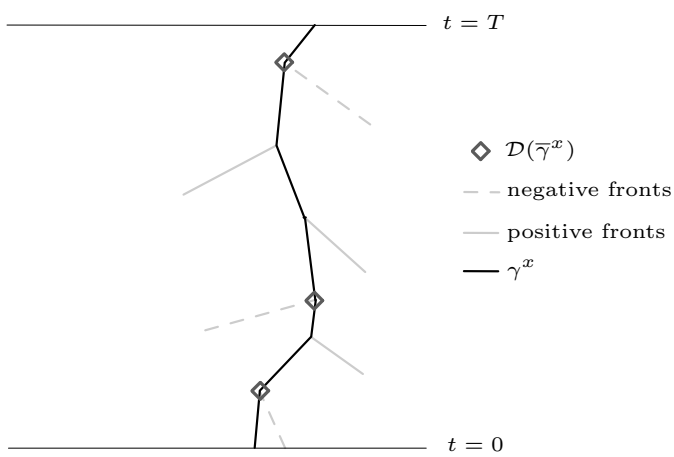

Figure 3: A example of positive $\gamma^{x}$

- Now we turn to the right front path $\gamma^{y}$, and prove (52). Here, we define

$$
\sigma^{y}(s):=\bar{\lambda}^{\max }\left(\gamma^{y}(s)^{-}, \gamma^{y}(s)^{+}\right)
$$

the maximal speed in the Riemann problem determined by the front $\gamma^{y}$ at time $s$. Again we get from the algorithm of Paragraph 4.2.1 and Lemmas 2 (see in particular (40)) and 3 that:

- if the interaction located at $\gamma^{y}(s)$ is monotone, then

$$
\sigma^{y}\left(s^{+}\right) \leq \sigma^{y}\left(s^{-}\right),
$$

- and if it is non-monotone, then

$$
\sigma^{y}\left(s^{+}\right) \leq \sigma^{y}\left(s^{-}\right)+\frac{\left\|\left(f^{\prime \prime}\right)-\right\|_{\infty}}{2} \Delta T V\left(\gamma^{x}(s)\right) .
$$

(Again the above inequality is valid whether the point $P:=\gamma^{x}(s)$ uses the splitting strategy or not, since the value $\bar{\lambda}^{\max }$ increases when one considers the outgoing fronts from left to right, and equals $\bar{\lambda}^{\max }\left(u_{l}^{P}, u_{r}^{P}\right)$ for the rightmost front.)

Now we note that for all $s$,

$$
\dot{\gamma}^{y}-\varepsilon \leq \sigma^{y}(s) \leq \dot{\gamma}^{y}+2 \varepsilon .
$$

This follows from the fact that all fronts have an inner speed variation less than $2 \varepsilon$ and from the convention of Paragraph 2.2. Hence summing (57) along all interactions that concern $\gamma^{y}$, and then applying (58), we get (52) in that case, where again $\mathcal{D}\left(\bar{\gamma}^{y}\right)$ is the set of non monotone interaction points on $\gamma^{y}$ during times in $(\tilde{t}, T)$. Again $\mathcal{N}$ is equal to 1 on it. 


\subsubsection{Proof of (51)-(52): negative front paths.}

We consider here the case where a front path selected above is negative during the whole time interval $[0, T]$. (Recall that in that case $\tilde{t}=0$.)

Considering the algorithm described in Paragraph 4.2, we see that during the history of this path, all interaction points are monotone (otherwise, the front path is either stopped or continued as a positive front). In particular, these points use the no splitting strategy.

We will classify these interaction points according to the following cases of the postprocessing algorithm:

- Class $a$ : points at which both incoming fronts were in the tree, and we extended $\gamma^{x}$ (or $\gamma^{y}$ ) by picking the incoming front on the right,

- Class $b$ : points at which both incoming fronts were in the tree, and we picked the one on the left,

- Class $c$ : points at which only one of the incoming fronts was in the tree.

For $z=x$ (resp. for $z=y$ ), call $\mathcal{I}_{a}^{z}, \mathcal{I}_{b}^{z}$ and $\mathcal{I}_{c}^{z}$ the set of interaction points of class $a, b$ and $c$, respectively, in the history of $\gamma^{x}$ (resp. in $\gamma^{y}$ ). We claim that for $t \in[0, T]$,

$$
\begin{aligned}
& f^{\prime}\left(\gamma^{x}(t)^{-}\right) \leq f^{\prime}\left(\gamma^{x}(T)^{-}\right)+\sum_{P \in \mathcal{I}_{a}^{x}} \tau\left(u_{l}^{P} ; u_{m}^{P}\right), \\
& f^{\prime}\left(\gamma^{y}(t)^{+}\right) \geq f^{\prime}\left(\gamma^{y}(T)^{+}\right)-\sum_{P \in \mathcal{I}_{b}^{y}} \tau\left(u_{m}^{P} ; u_{r}^{P}\right) .
\end{aligned}
$$

Let us explain this in the case (59); (60) is treated likewise. We follow $\gamma^{x}$ (backward in time): the characteristic speeds on the sides of $\gamma^{x}$ change only at interaction times. We want to compare the characteristic speed of the state on the left of $\gamma^{x}$ before and after the interaction. We discuss according to the class of the interaction point:

- Class $a$ : in that case, as we go across the interaction in growing time, the left state is changed and its characteristic speed has decreased of $\tau\left(u_{l}^{P} ; u_{m}^{P}\right)>0$ (since the left incoming front was in the tree, it has a positive osv). This is taken into account in the last term of (59).

- Class $b$ : in that case, as we go across the interaction in growing time, the left state is conserved, so there is nothing to add to (59).

- Class $c$ : in that that case, the outer speed variation of the front that we did not pick in the tree was nonpositive. Hence,

- either it was the left incoming front, and the left characteristic speed has increased (or has stayed constant),

- either it was the right incoming front, and the left state is conserved.

In both cases, we do not need to add an error term to (59).

Hence we get (59)-(60)

Now we claim that the terms $\sum_{P \in \mathcal{I}_{a}^{x}} \tau\left(u_{l}^{P} ; u_{m}^{P}\right)$ and $\sum_{P \in \mathcal{I}_{b}^{y}} \tau\left(u_{m}^{P} ; u_{r}^{P}\right)$ can be estimated in the total variation dissipation term $\sqrt{\left\|\left(f^{\prime \prime}\right)_{-}\right\|_{\infty}\left\|f^{\prime \prime}\right\|_{\infty}}\left(T V\left(u_{0}\right)-T V(u(t))\right)$. This is a consequence of Lemmas 5 and 6 . The main fact is that the front that has been left in cases $a$ for the $x$-tree and $b$ for the $y$-tree has a good osv source tree. We explain this in the case of the $x$-tree, since the reasoning is the same in the case of the $y$-tree. Consider an interaction point counted in $\mathcal{I}_{a}^{x}$. We consider the incoming front that is not selected in $\gamma^{x}$ at this point (that is, $\left(u_{l}^{P} ; u_{m}^{P}\right)$ since $\left.P \in \mathcal{I}_{a}^{x}\right)$, and draw the extended osv source tree of this front. We claim that this tree is good (in the sense of Paragraph 3.4). Indeed, if one of its leaves were not good, then it follows from the construction of Paragraph 4.2.2 (see Remark 7) that this osv source tree had a branch touching $t=0$, which is in contradiction with the fact that we are in case $a$ (we did not pick the front $\left(u_{l}^{P} ; u_{m}^{P}\right)$ when applying the postprocessing algorithm of Paragraph 4.2.3).

Now, using (59)-(60), and Lemma 6, we arrive at

$$
\begin{aligned}
& f^{\prime}\left(\gamma^{x}(t)^{-}\right) \leq f^{\prime}\left(\gamma^{x}(T)^{-}\right)+\sum_{P \in \mathcal{I}_{a}^{x}} \sum_{\beta \in \widehat{\mathcal{L}}\left(u_{l}^{P} ; u_{m}^{P}\right)} \vartheta(\beta), \\
& f^{\prime}\left(\gamma^{y}(t)^{+}\right) \geq f^{\prime}\left(\gamma^{y}(T)^{+}\right)-\sum_{P \in \mathcal{I}_{b}^{y}} \sum_{\beta \in \widehat{\mathcal{L}}\left(u_{m}^{P} ; u_{r}^{P}\right)} \vartheta(\beta) .
\end{aligned}
$$

Now we will use Lemma 5 to estimate each $\vartheta(\beta)$, but we do not apply directly Lemma 5 to the front $\beta$. From the construction, the front $\beta$ outgoes from a non-monotone interaction point $Q$ at $t>0$ which uses the splitting 
strategy; it is not the leftmost (resp. the rightmost) outgoing front, if $u_{r}^{Q}<u_{l}^{Q}<u_{m}^{Q}$ (resp. if $u_{m}^{Q}<u_{r}^{Q}<u_{l}^{Q}$ ). Again we call $\mu_{1}, \ldots, \mu_{\nu_{Q}}$, the fronts outgoing from $Q$. We study the cases $u_{r}^{Q}<u_{l}^{Q}<u_{m}^{Q}$ and $u_{m}^{Q}<u_{r}^{Q}<u_{l}^{Q}$ separately.

- First case: $u_{r}^{Q}<u_{l}^{Q}<u_{m}^{Q}$. In that case, it follows from Proposition 4-(28) that

$$
\vartheta\left(u_{l}^{Q} ; u_{r}^{Q}\right) \leq \vartheta\left(u_{m}^{Q} ; u_{r}^{Q}\right)+\sqrt{\left\|\left(f^{\prime \prime}\right)_{-}\right\|_{\infty}\left\|f^{\prime \prime}\right\|_{\infty}} \Delta T V(Q) .
$$

Now we apply Lemma 5 on the incoming front $\left(u_{m}^{Q} ; u_{r}^{Q}\right)$ to get

$$
\vartheta\left(u_{m}^{Q} ; u_{r}^{Q}\right)-\varepsilon \leq \sqrt{\left\|\left(f^{\prime \prime}\right)-\right\|_{\infty}\left\|f^{\prime \prime}\right\|_{\infty}} \sum_{R \in \mathcal{V}\left(u_{m}^{Q} ; u_{r}^{Q}\right)} \Delta T V(R) .
$$

Recalling the construction of the approximate solver with the splitting strategy, and in particular (19) (see also Remark 3), we deduce

$$
\sum_{i=2}^{\nu_{Q}} \vartheta\left(\mu_{i}\right)=\vartheta\left(u_{l}^{Q} ; u_{r}^{Q}\right)-\varepsilon
$$

and hence that

$$
\sum_{i=2}^{\nu_{Q}} \vartheta\left(\mu_{i}\right) \leq \sqrt{\left\|\left(f^{\prime \prime}\right)-\right\|_{\infty}\left\|f^{\prime \prime}\right\|_{\infty}}\left(\Delta T V(Q)+\sum_{R \in \mathcal{V}\left(u_{m}^{Q} ; u_{r}^{Q}\right)} \Delta T V(R)\right)
$$

(Note that there are at least three outgoing fronts when the splitting strategy is applied apart from $t=0$.) In other words, we can measure by a term of the type

$$
\sqrt{\left\|\left(f^{\prime \prime}\right)-\right\|_{\infty}\left\|f^{\prime \prime}\right\|_{\infty}} \times(\text { total variation dissipation })
$$

the inner speed variation of all the outgoing fronts at $Q$, except the leftmost one. But recall from Paragraph 4.2.2 that this latter front will not stay a purely negative one, so it does not have to be counted here. In that case, we fix $\tilde{\iota}_{\beta}:=\iota_{\beta}-1$; this will be used later.

- Second case: $u_{m}^{Q}<u_{r}^{Q}<u_{l}^{Q}$. This case is treated exactly as the previous one: replace the use of (28) with the one of (27); apply Lemma 5 to $\left(u_{l}^{Q} ; u_{m}^{Q}\right)$ and finally arrive at

$$
\sum_{i=1}^{\nu_{Q}-1} \vartheta\left(\mu_{i}\right) \leq \sqrt{\left\|\left(f^{\prime \prime}\right)-\right\|_{\infty}\left\|f^{\prime \prime}\right\|_{\infty}}\left(\Delta T V(Q)+\sum_{R \in \mathcal{V}\left(u_{l}^{Q} ; u_{m}^{Q}\right)} \Delta T V(R)\right) .
$$

(Here the rightmost outgoing front is excluded.) In that case, we fix $\tilde{\iota}_{\beta}:=\iota_{\beta}$.

Now we choose $\mathcal{D}\left(\bar{\gamma}^{x}\right)$ and $\mathcal{D}\left(\bar{\gamma}^{y}\right)$ as follows. We summarize the situation: for each $P \in \mathcal{I}_{a}^{x}$ (resp. $P \in \mathcal{I}_{b}^{y}$ ), we associate the leaves $\beta$ of the osv source tree of the left (resp. right) incoming front at $P$; to each leaf $\beta$ we associate the interaction point $Q$ from which $\beta$ is outgoing; to this $Q$ we associate the vertices of the isv source tree of the negative incoming front at $Q$; we denote these interaction points by $R$. Now we define $\mathcal{D}\left(\bar{\gamma}^{x}\right)(\operatorname{resp}$. $\left.\mathcal{D}\left(\bar{\gamma}^{y}\right)\right)$ as the union of $\left(R, \tilde{\iota}_{\beta}\right)$ for all of these interaction points $R$, together with $\left(Q, \tilde{\iota}_{\beta}\right)$. (The "- 1 " in the above $\tilde{\iota}_{\beta}:=\iota_{\beta}-1$ is intended to make the marking consistent with the second union in (50), which begins at $i=1$.)

We illustrate this in Figure 4 below: the solid lines represent the osv source tree of $\bar{\gamma}^{x}$, the black solid lines being $\gamma^{x}$ itself; the dotted lines are fronts that do not belong to any tree; the dashed lines represent the isv sources trees.

We observe that in the above construction, each $(R, i)$ is associated to a unique $(Q, i): Q$ is the first splitting point that we meet when we follow the fronts in the approximation forward in time, starting from $R$. In turn, each $(Q, i)$ is associated to a unique $\beta$ given by the $i$-th outgoing front at $Q$ (or the $(i+1)$-th one, according to the nature of the interaction at $Q$ ); each $\beta$ is associated to a unique $P \in \mathcal{I}_{a}^{x}$ or $P \in \mathcal{I}_{b}^{y}$ : $P$ is the first point in $\gamma^{x}$ or $\gamma^{y}$ that we meet when going forward in time in the approximation, starting from $\beta$. In other words, each "marked interaction point" is counted at most once in the above sums.

Transferring the previous information into (61)-(62), we obtain

$$
\begin{aligned}
& f^{\prime}\left(\gamma^{x}(t)^{-}\right) \leq f^{\prime}\left(\gamma^{x}(T)^{-}\right)+\sqrt{\left\|\left(f^{\prime \prime}\right)-\right\|_{\infty}\left\|f^{\prime \prime}\right\|_{\infty}} \sum_{(M, i) \in \mathcal{D}\left(\bar{\gamma}^{x}\right)} \Delta T V(M, i), \\
& f^{\prime}\left(\gamma^{y}(t)^{+}\right) \geq f^{\prime}\left(\gamma^{y}(T)^{+}\right)-\sqrt{\left\|\left(f^{\prime \prime}\right)_{-}\right\|_{\infty}\left\|f^{\prime \prime}\right\|_{\infty}} \sum_{(M, i) \in \mathcal{D}\left(\bar{\gamma}^{y}\right)} \Delta T V(M, i) .
\end{aligned}
$$

Finally, as by construction, for all $t \in[0, T]$, the front $\gamma^{x}(t)$ (resp. $\left.\gamma^{y}(t)\right)$ has a positive outer speed variation and an inner speed variation no more than $2 \varepsilon$, we have

$$
f^{\prime}\left(\gamma^{z}(t)^{+}\right) \leq \bar{\lambda}^{\max }\left(\gamma^{z}(t)^{-} ; \gamma^{z}(t)^{+}\right) \leq \bar{\lambda}^{\min }\left(\gamma^{z}(t)^{-} ; \gamma^{z}(t)^{+}\right)+2 \varepsilon \leq f^{\prime}\left(\gamma^{z}(t)^{-}\right)+2 \varepsilon \leq f^{\prime}\left(\gamma^{z}(t)^{+}\right)+2 \varepsilon,
$$

for $z=x$ or $y$, which yields (51)-(52) in that case. 


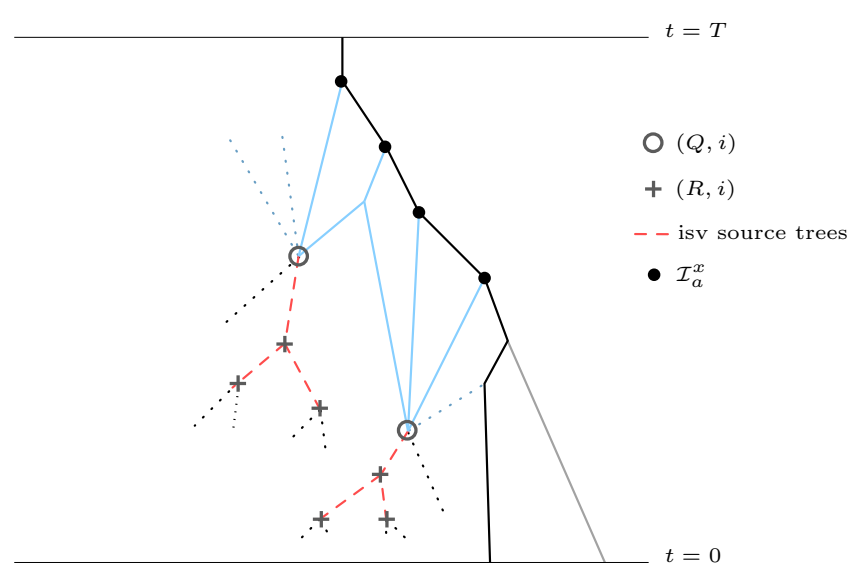

Figure 4: A example of negative $\gamma^{x}$

\subsubsection{Proof of (51)-(52): positive-negative front paths.}

Now we consider a front path that is successively positive and negative. According to the algorithm described in Paragraph 4.1, there is some $\hat{t} \in(0, T)$ such that the front under view is positive during $[0, \hat{t})$, and then negative during $(\hat{t}, T]$. As the front paths that we select do not meet when they are negative, we have $\tilde{t}<\hat{t}$.

Performing the above reasonings during time intervals $[\tilde{t}, \hat{t}]$ (Paragraph 4.3.3) and $[\hat{t}, T]$ (Paragraph 4.3.4), we see that it is enough to compare $\dot{\gamma}^{x}\left(\hat{t}^{-}\right)$and $\dot{\gamma}^{x}\left(\hat{t}^{+}\right)$(resp. $\dot{\gamma}^{y}\left(\hat{t}^{-}\right)$and $\left.\dot{\gamma}^{y}\left(\hat{t}^{+}\right)\right)$and to show that the error is of order $\varepsilon$. But according to the algorithm of Paragraph 4.2.2, we see that the situation where a negative front is continued as a positive front occurs only if the interaction point at time $\hat{t}$ is a non-monotone interaction point $P$ (with negative outgoing fronts), and we are in one of the following three cases:

- or the no-splitting strategy was used,

- or $u_{r}^{P}<u_{l}^{P}<u_{m}^{P}$ and the outgoing front that we considered was the leftmost one,

- or $u_{m}^{P}<u_{r}^{P}<u_{l}^{P}$ and the outgoing front that we considered was the rightmost one.

Note that the first case is in fact included in the two latter ones. Moreover, the negative outgoing front $\gamma^{x}\left(\hat{t}^{+}\right)$ or $\gamma^{y}\left(\hat{t}^{+}\right)$under view has a positive outer speed variation by construction. Hence, one can apply Lemma 4 , with in both cases the term within brackets equal to zero. One easily concludes that

$$
\left|\dot{\gamma}^{z}\left(\hat{t}^{-}\right)-\dot{\gamma}^{z}\left(\hat{t}^{+}\right)\right| \leq 4 \varepsilon
$$

in both cases $z=x$ and $z=y$.

Hence we can define $\mathcal{D}\left(\bar{\gamma}^{x}\right)$ (resp. $\mathcal{D}\left(\bar{\gamma}^{y}\right)$ ) by taking the union of the representatives $(M, i)$ selected for the negative fronts during times $(\hat{t}, T]$ (following Paragraph 4.3.4) and of the representatives $(M, 1)$ selected for the positive fronts during times [0, $\hat{t}$ ) (following Paragraph 4.3.3), see an example in Figure 5. Remark in particular that in Lemma 4, no total variation dissipation is used; consequently we do not add a representative of $\gamma^{x}(\hat{t})$ to the selection $\mathcal{D}\left(\bar{\gamma}^{x}\right)\left(\right.$ or $\left.\mathcal{D}\left(\bar{\gamma}^{y}\right)\right)$.

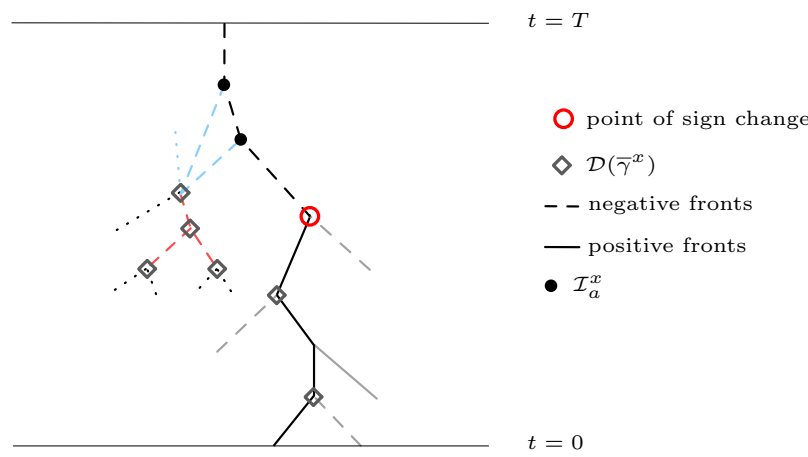

Figure 5: A example of positive-negative $\gamma^{x}$ 


\subsubsection{Proof of (53).}

For $\alpha \notin \mathcal{A} \cup\left\{\bar{\gamma}^{x}, \bar{\gamma}^{y}\right\}$, we can define $\mathcal{D}(\alpha):=\emptyset$, since these fronts have no importance. This includes positive fronts at time $T$ which do not belong to $\left\{\bar{\gamma}^{x}, \bar{\gamma}^{y}\right\}$, since abandoned fronts are negative.

Now let us define $\mathcal{D}(\alpha)$ for $\alpha \in \mathcal{A}$. There are several cases to consider, according to the reason why the algorithm abandoned $\alpha$ :

- if $\tau(\alpha) \leq 0$, define $\mathcal{D}(\alpha):=\emptyset$, in such a way that (53) is satisfied, and that this front will not have to be considered in the proof of (54).

- if $\tau(\alpha)>0$, we reason in the same way as we did in Paragraph 4.3 .4 concerning fronts $\left(u_{l}^{P} ; u_{m}^{P}\right)$ with $P \in \mathcal{I}_{a}^{x}$. We begin by drawing the osv source tree of $\alpha$. The fact that $\alpha$ was abandoned implies that this tree is good. As in Paragraph 4.3.4, we associate to each of its leaves $\beta$ the corresponding interaction point $Q$ from which this leaf outgoes. Call $\iota_{\beta}$ the rank of $\beta$ as a front outgoing from $Q$, and determine $\tilde{\iota}_{\beta}$ as Paragraph 4.3.4. Now we consider the "larger" incoming front at $Q$ (that is the negative one), and the isv source tree of this larger front. We define $\mathcal{D}(\alpha)$ as the set of the vertices of this isv source tree, where we add $\tilde{\iota}_{\beta}$ as a marker, plus $\left(Q, \tilde{\iota}_{\beta}\right)$. Now, proceeding as in Paragraph 4.3 .4 , we get that

$$
\vartheta(\beta) \leq \sqrt{\left\|\left(f^{\prime \prime}\right)-\right\|_{\infty}\left\|f^{\prime \prime}\right\|_{\infty}}\left(\Delta T V\left(Q, \tilde{\iota}_{\beta}\right)+\sum_{R \in \mathcal{V}\left(u_{-}^{Q} ; u_{+}^{Q}\right)} \Delta T V\left(R, \tilde{\iota}_{\beta}\right)\right),
$$

where $\left(u_{-}^{Q} ; u_{+}^{Q}\right)$ is the bigger incoming front at $Q$. Hence (53) follows from Lemma 6 as previously.

\subsubsection{Proof of (54).}

It remains to establish (54). Consider a representative $(M, i)$ of an interaction point $M$ which belongs to $\mathcal{D}(\alpha) \cap$ $\mathcal{D}(\beta)$, where $\alpha$ and $\beta$ are two different fronts living at time $T$. Several cases are in order:

- if the front(s) outgoing from $M$ is (are) positive, then necessarily $(M, i)=(M, 1)$ has been selected in $\mathcal{D}(\alpha)$ and $\mathcal{D}(\beta)$ during Paragraph 4.3 .3 or during the "positive part" of Paragraph 4.3.5, since the other representatives $(M, i)$ which are selected in a set $\mathcal{D}$ have negative outgoing fronts. In particular, it is not possible for $\alpha$ or $\beta$ to be an abandoned front. Hence $\{\alpha, \beta\}=\left\{\bar{\gamma}^{x}, \bar{\gamma}^{y}\right\}$. But for positive front paths, we only consider $(M, i)$ inside $\gamma^{x}$ or $\gamma^{y}$, (strictly) after time $\tilde{t}$; this contradicts the definition of $\tilde{t}$.

- if the front(s) outgoing from $M$ is negative: there are two types of such $(M, i)$ : those of type $(Q, i)$ where $Q$ is a splitting point which has been selected as the bottom of the leaf of an osv source tree; those of type $(R, i)$ where $R$ has been selected as the vertex of an isv source tree. As noticed above, these are easy to distinguish, because the first type $(Q, i)$ corresponds to a splitting point, while the latter type of $(R, i)$ corresponds to a non splitting point. We treat these two types separately.

- $M$ is non splitting. Starting from $M$, we follow the fronts forward in time until we reach a splitting interaction point $Q$. This is done in a clear unique way. It follows from the construction that for both $\mathcal{D}(\alpha)$ and $\mathcal{D}(\beta), Q$ is an interaction point from which outgoes a leaf $\mu$ of an osv source tree drawn from either $\alpha, \beta \in \mathcal{A}$ or from $P \in \mathcal{I}_{a}^{x} \cup \mathcal{I}_{b}^{y}$. Moreover, the marker $i$ of $(M, i)$ indicates which one of the outgoing fronts at $Q$ is $\mu$.

In the case where $\mu$ is constructed from the osv source tree of $P \in \mathcal{I}_{a}^{x} \cup \mathcal{I}_{b}^{y}$, the point $P$ itself was constructed as a vertex of the osv source tree of $\bar{\gamma}^{x}$ (resp. $\bar{\gamma}^{y}$ ). From the transitivity of Lemma 6 , it follows that $\mu$ belongs to the osv source tree of $\bar{\gamma}^{x}$ (resp. $\bar{\gamma}^{y}$ ). In all cases, we see that $\mu$ belongs to $\widehat{\mathcal{T}}(\alpha) \cap \widehat{\mathcal{T}}(\beta)$. Now the fact that the osv source trees are disjoint in the sense of Lemma 6 implies that $\alpha=\beta$, since osv source trees can have only one front living at time $T$.

- $M$ is splitting. We can reason as for the point $Q$ in the above case and conclude in the same way.

This ends the proof of Proposition 5.

\subsection{Conclusion}

There remain three steps to establish (11):

- to obtain a form of (49) which does not count the cost of abandoned trees (yet) in the two following cases:

- Case 1: $\gamma^{x}$ and $\gamma^{y}$ do not meet except perhaps at $t=0$,

- Case 2: $\gamma^{x}$ and $\gamma^{y}$ meet at a time $\tilde{t}>0$,

- to count the cost of abandoned trees on (49) (and to treat the case $\bar{\gamma}^{x}=\bar{\gamma}^{y}$ ),

- to pass to the limit in (49) in order to obtain (11).

This is done in the next paragraphs. 


\subsubsection{Case 1}

Suppose that we are in the first situation. This is described in Figure 6. We have

$$
\begin{aligned}
\bar{\gamma}^{y}-\bar{\gamma}^{x} & =\gamma^{y}(0)-\gamma^{x}(0)+\int_{0}^{T}\left[\dot{\gamma}^{y}(\tau)-\dot{\gamma}^{x}(\tau)\right] d \tau \\
& \geq \int_{0}^{T}\left[\dot{\gamma}^{y}(\tau)-\dot{\gamma^{x}}(\tau)\right] d \tau,
\end{aligned}
$$

since the fronts $\gamma^{x}$ and $\gamma^{y}$ did not cross each other. Now, using Proposition 5, we get that

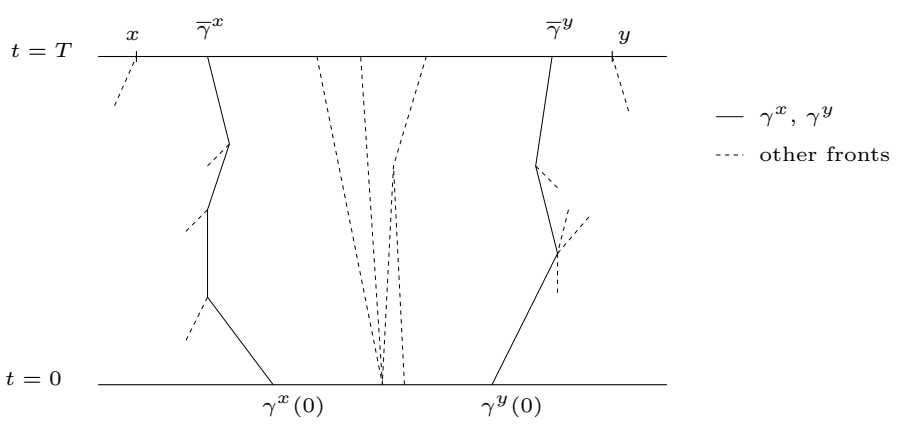

Figure 6: Case 1

$$
y-x \geq \bar{\gamma}^{y}-\bar{\gamma}^{x} \geq T\left(\dot{\gamma^{y}}(T)-\gamma^{x}(T)\right)-\sqrt{\left\|\left(f^{\prime \prime}\right)_{-}\right\|_{\infty}\left\|f^{\prime \prime}\right\|_{\infty}} T[\widetilde{\Delta} T V(T, x, y)]-16 \varepsilon T .
$$

where $\widetilde{\Delta} T V(T, x, y)$ is a part of the total variation dissipation:

$$
\widetilde{\Delta} T V(T, x, y):=\sum_{(P, i) \in \mathcal{D}\left(\bar{\gamma}^{x}\right)} \Delta T V(P, i)+\sum_{(P, i) \in \mathcal{D}\left(\bar{\gamma}^{y}\right)} \Delta T V(P, i) \leq T V\left(u_{0}\right)-T V(u(T)) .
$$

Now the construction and the admissibility of the solutions of the Riemann problems $\left(u\left(\bar{\gamma}^{x-}\right) ; u\left(\bar{\gamma}^{x+}\right)\right)$ and $\left(u\left(\bar{\gamma}^{y-}\right) ; u\left(\bar{\gamma}^{y+}\right)\right)$ involve that

$$
f^{\prime}\left(u\left(\bar{\gamma}^{x-}\right)\right) \geq \dot{\gamma^{x}}(T)-\varepsilon \text { and } f^{\prime}\left(u\left(\bar{\gamma}^{y+}\right)\right) \leq \dot{\gamma}^{y}(T)+2 \varepsilon .
$$

Together with (65)-(66), this yields the conclusion.

\subsubsection{Case 2}

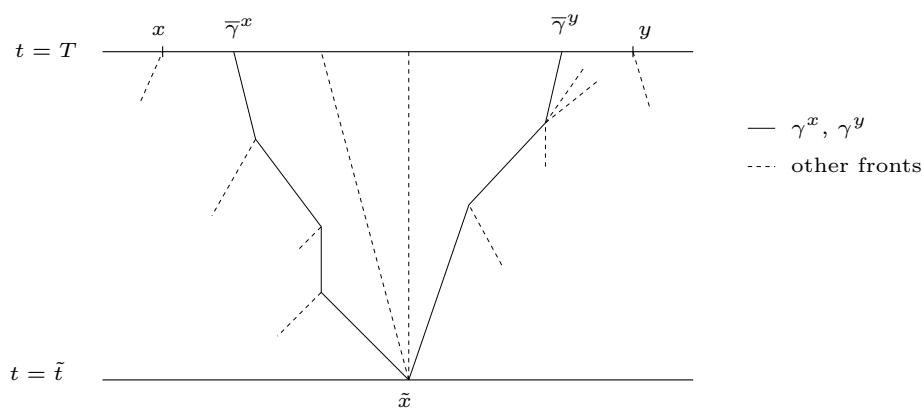

Figure 7: Case 2

Now we suppose that we are in the second situation, such as described in Figure 7 . We call $\tilde{t}>0$ the time of meeting of $\gamma^{x}$ and $\gamma^{y}$, and denote $\tilde{x}:=\gamma^{x}(\tilde{t})=\gamma^{y}(\tilde{t})$. Recall that $\gamma^{x}$ and $\gamma^{y}$ are necessarily positive fronts at the time they meet. Arguing as previously, we get that

$$
\begin{aligned}
f^{\prime}\left(u\left(\bar{\gamma}^{y+}\right)\right)-f^{\prime}\left(u\left(\bar{\gamma}^{x-}\right)\right) & \leq \dot{\gamma}^{y}(T)-\dot{\gamma}^{x}(T)+3 \varepsilon \\
& \leq \dot{\gamma}^{y}(\tilde{t})-\dot{\gamma}^{x}(\tilde{t})+\sqrt{\left\|\left(f^{\prime \prime}\right)_{-}\right\|_{\infty}\left\|f^{\prime \prime}\right\|_{\infty}} \widetilde{\Delta} T V(T, x, y)+\mathcal{O}(\varepsilon) .
\end{aligned}
$$


Thus, if we manage to prove that

$$
\dot{\gamma}^{y}(\tilde{t})-\dot{\gamma}^{x}(\tilde{t}) \leq \sqrt{\left\|\left(f^{\prime \prime}\right)_{-}\right\|_{\infty}\left\|f^{\prime \prime}\right\|_{\infty}} \Delta T V(\tilde{t}, \tilde{x})+\mathcal{O}(\varepsilon),
$$

we will have established (49) (even, without the non-negative $(y-x) / T$ term). Recall in particular that the interaction point at time $\tilde{t}$ is not included in $\mathcal{D}\left(\bar{\gamma}^{x}\right)$ and $\mathcal{D}\left(\bar{\gamma}^{y}\right)$ (see Paragraph 4.3 .3 concerning positive front paths). Moreover this interaction point cannot appear in other sets $\mathcal{D}$ since it has positive outgoing fronts.

Since there are several fronts outgoing from $(\tilde{t}, \tilde{x})$, the interaction taking place at $(\tilde{t}, \tilde{x})$ is necessarily nonmonotone (and uses the splitting strategy). Hence, using Proposition 4, it follows that in the outgoing Riemann problem $\left(u\left(\tilde{t}, \tilde{x}^{-}\right) ; u\left(\tilde{t}, \tilde{x}^{+}\right)\right)$the inner speed variation satisfies:

$$
\vartheta\left(u\left(\tilde{t}, \tilde{x}^{-}\right) ; u\left(\tilde{t}, \tilde{x}^{+}\right)\right) \leq 2 \varepsilon+\sqrt{\left\|\left(f^{\prime \prime}\right)_{-}\right\|_{\infty}\left\|f^{\prime \prime}\right\|_{\infty}} \Delta T V(\tilde{t}, \tilde{x}) .
$$

But it follows from the construction of the solver using the splitting strategy that

$$
\bar{\lambda}^{\min }\left(\gamma^{y}\left(\tilde{t}^{+}\right)^{-} ; \gamma^{y}\left(\tilde{t}^{+}\right)^{+}\right)-\bar{\lambda}^{\min }\left(\gamma^{x}\left(\tilde{t}^{+}\right)^{-} ; \gamma^{x}\left(\tilde{t}^{+}\right)^{+}\right) \leq \vartheta\left(u\left(\tilde{t}, \tilde{x}^{-}\right) ; u\left(\tilde{t}, \tilde{x}^{+}\right)\right) .
$$

Taking the possible additional displacement of fronts of Paragraph 2.2 into account, we deduce

$$
\dot{\gamma}^{y}\left(\tilde{t}^{+}\right)-\dot{\gamma}^{x}\left(\tilde{t}^{+}\right) \leq \vartheta\left(u\left(\tilde{t}, \tilde{x}^{-}\right) ; u\left(\tilde{t}, \tilde{x}^{+}\right)\right)+\varepsilon .
$$

Hence, (67) follows, which concludes the proof of (49) where the abandoned trees still have to be counted.

\subsubsection{The cost of abandoned trees}

At this step we have proved that

$$
f^{\prime}\left(\left(\bar{\gamma}^{y}\right)^{+}\right)-f^{\prime}\left(\left(\bar{\gamma}^{x}\right)^{-}\right) \leq \frac{y-x}{T}+\sqrt{\left\|\left(f^{\prime \prime}\right)_{-}\right\|_{\infty}\left\|f^{\prime \prime}\right\|_{\infty}} \sum_{(M, i) \in \mathcal{D}\left(\bar{\gamma}^{x}\right) \cup \mathcal{D}\left(\bar{\gamma}^{x}\right)} \Delta T V(M, i)+\mathcal{O}(\varepsilon) .
$$

Note that the case when the process of Paragraph 4.2.3 made $\bar{\gamma}^{x}=\bar{\gamma}^{y}$ can be included in that case. Indeed it is again a consequence of (25) and (36) that

$$
f^{\prime}\left(\bar{\gamma}^{x+}\right)-f^{\prime}\left(\bar{\gamma}^{x-}\right) \leq 2 \varepsilon
$$

Hence (68) is still valid in this case by setting $\mathcal{D}\left(\bar{\gamma}^{x}\right)=\mathcal{D}\left(\bar{\gamma}^{y}\right)=\emptyset$.

It remains to justify why the trees that we abandoned in the process of Paragraph 4.2 .3 can be counted in the "rest" of the total variation dissipation term $\sqrt{\left\|\left(f^{\prime \prime}\right)_{-}\right\|_{\infty}\left\|f^{\prime \prime}\right\|_{\infty}}\left[T V\left(u_{0}\right)-T V(u(t))\right]$. There are two kinds of abandoned fronts:

- those that were abandoned because they had a negative osv: jumping them makes the inequality to prove stronger,

- those were abandoned because their osv source tree did not reach $t=0$, but which had a positive osv: the error made in (49) by jumping to the other side of the front is precisely measured by Proposition 5-(53), hence this can be incorporated in $\sqrt{\left\|\left(f^{\prime \prime}\right)_{-}\right\|_{\infty}\left\|f^{\prime \prime}\right\|_{\infty}}\left[T V\left(u_{0}\right)-T V(u(t))\right]$.

In other words,

$$
\begin{aligned}
f^{\prime}(y)-f^{\prime}(x)-\left[f^{\prime}\left(\bar{\gamma}^{y+}\right)-f^{\prime}\left(\bar{\gamma}^{x-}\right)\right] & =\sum_{\alpha \in \mathcal{A}} \tau(\alpha) \\
& \leq \sqrt{\left\|\left(f^{\prime \prime}\right)-\right\|\left\|_{\infty}\right\| f^{\prime \prime} \|_{\infty}} \sum_{(M, i) \in \sum_{\alpha \in \mathcal{A}} \mathcal{D}(\alpha)} \Delta T V(M, i) .
\end{aligned}
$$

This concludes the proof of (49).

\subsubsection{Proof of (11)}

It remains to pass to the limit in (49). Remark that for each $t \in \mathbb{R}^{+}$, due to (30), one has the convergence (up to a subsequence),

Now using (24) and

$$
u_{\varepsilon_{\nu}}(t, x) \rightarrow u(t, x) \text { for almost every } x \in \mathbb{R} \text {. }
$$

$$
T V(u(t, \cdot)) \leq \liminf _{\nu \rightarrow+\infty} T V\left(u_{\varepsilon_{\nu}}(t, \cdot)\right)
$$


we reach the conclusion that

$$
f^{\prime}(u(t, y))-f^{\prime}(u(t, x)) \leq \frac{y-x}{t}+\sqrt{\left\|\left(f^{\prime \prime}\right)_{-}\right\|_{\infty}\left\|f^{\prime \prime}\right\|_{\infty}}[T V(u(0, \cdot))-T V(u(t, \cdot))] \text { for a. e. } x \leq y .
$$

Since $u$ has a representative such that $u(t, \cdot)$ is left continuous in $\mathbb{R}$ for all $t$ (see e.g. [1, Theorem 2.4]), we conclude that, if we modify $u$ on some negligible set, (69) is valid everywhere, which concludes the proof of (11) when $u_{0} \in L^{1}(\mathbb{R}) \cap B V(\mathbb{R})$.

Of course, the general case $u_{0} \in L^{\infty}(\mathbb{R}) \cap B V_{\text {loc }}(\mathbb{R})$ follows easily by using the finite speed of propagation: it suffices modify properly $u_{0}$ (bringing it back 0 for instance) outside a zone $[x-\hat{\lambda} T, y+\hat{\lambda} T]$, where $\hat{\lambda}>$ $\left\|f^{\prime}\right\|_{L^{\infty}\left(\left[-\left\|u_{0}\right\|_{\infty} ;\left\|u_{0}\right\|_{\infty}\right]\right)}$.

\section{$5 \quad$ Proof of the technical results}

In this section, we establish all the technical results mentioned above.

\subsection{Proof of the interaction estimates on the speeds.}

\subsubsection{Proof of Lemma 2 .}

We only treat the case of $u_{l}<u_{m}<u_{r}$, since the case $u_{l}>u_{m}>u_{r}$ is entirely similar.

- Concerning (37): this is a direct consequence of $\operatorname{conv}_{\left[u_{l}, u_{r}\right]} f \leq \operatorname{conv}_{\left[u_{l}, u_{m}\right]} f$ on $\left[u_{l}, u_{m}\right]$.

- In the same way, inequality (38) is consequence of $\operatorname{conv}_{\left[u_{l}, u_{r}\right]} f \leq \operatorname{conv}_{\left[u_{m}, u_{r}\right]} f$ on $\left[u_{m}, u_{r}\right]$.

- Now, to establish (39): let us suppose that the first possibility in (39) is not satisfied, that is, $\bar{\lambda}^{\min }\left(u_{l} ; u_{r}\right)<$ $\bar{\lambda}^{\min }\left(u_{l} ; u_{m}\right)$. It follows that $\left(\operatorname{conv}_{\left[u_{l}, u_{r}\right]} f\right)$ has no contact point with $f$ in $\left(u_{l} ; u_{m}\right]$ : if it $\operatorname{did}\left(\operatorname{call} u^{*}\right.$ such a point), then it would follow that

$$
\left(\operatorname{conv}_{\left[u_{l}, u_{r}\right]} f\right)_{\mid\left[u_{l}, u^{*}\right]} \equiv\left(\operatorname{conv}_{\left[u_{l}, u_{m}\right]} f\right)_{\mid\left[u_{l}, u^{*}\right]},
$$

in contradiction with our assumption. Now we consider

$$
\bar{u}:=\min \left\{u \in\left[u_{m} ; u_{r}\right],\left(\operatorname{conv}_{\left[u_{l}, u_{r}\right]} f\right)(u)=f(u)\right\} .
$$

It follows that

$$
\left(\operatorname{conv}_{\left[u_{l}, u_{r}\right]} f\right)_{\mid\left[\bar{u}, u_{r}\right]} \equiv\left(\operatorname{conv}_{\left[u_{m}, u_{r}\right]} f\right)_{\mid\left[\bar{u}, u_{r}\right]},
$$

and that on $\left[u_{l}, \bar{u}\right], \operatorname{conv}_{\left[u_{l}, u_{r}\right]} f$ is affine. The conclusion follows easily that

$$
\bar{\lambda}^{\min }\left(u_{l} ; u_{r}\right)=\left(\operatorname{conv}_{\left[u_{l}, u_{r}\right]} f\right)^{\prime}(\bar{u}) \geq\left(\operatorname{conv}_{\left[u_{m}, u_{r}\right]} f\right)^{\prime}\left(u_{m}\right)=\bar{\lambda}^{\min }\left(u_{m} ; u_{r}\right) .
$$

- The proof of (40) is similar to the proof of (39): just consider

$$
\bar{u}:=\max \left\{u \in\left[u_{l} ; u_{m}\right],\left(\operatorname{conv}_{\left[u_{l}, u_{r}\right]} f\right)(u)=f(u)\right\}
$$

instead of (70).

\subsubsection{Proof of Lemma 3}

We have two inequalities to prove in the two cases of a non-monotone interaction which generate a positive outgoing wave. We prove these four properties separately.

- Inequality (41) in the case $u_{l}<u_{r}<u_{m}$. In that case (41) is again a consequence of the general property of the convex hull that

$$
\left(\operatorname{conv}_{[a, b]} f\right)^{\prime}(a) \geq\left(\operatorname{conv}_{[a, c]} f\right)^{\prime}(a) \text { for } a \leq b \leq c,
$$

which results from $\operatorname{conv}_{[a, b]} f \geq\left(\operatorname{conv}_{[a, c]} f\right)_{\mid[a, b]}$. Consequently the term $-\left(\left\|\left(f^{\prime \prime}\right)_{-}\right\|_{\infty} / 2\right) \Delta T V$ is actually not necessary here.

- Inequality (42) in the case $u_{l}<u_{r}<u_{m}$. In that case $\left(u_{-}, u_{+}\right)=\left(u_{l}, u_{m}\right)$ and $\Delta T V=u_{m}-u_{r}$. In terms of convex hulls, we have

$$
\bar{\lambda}^{\max }\left(u_{l} ; u_{r}\right)-\bar{\lambda}^{\max }\left(u_{-} ; u_{+}\right)=\left(\operatorname{conv}_{\left[u_{l}, u_{r}\right]} f\right)^{\prime}\left(u_{r}\right)-\left(\operatorname{conv}_{\left[u_{l}, u_{m}\right]} f\right)^{\prime}\left(u_{m}\right) .
$$




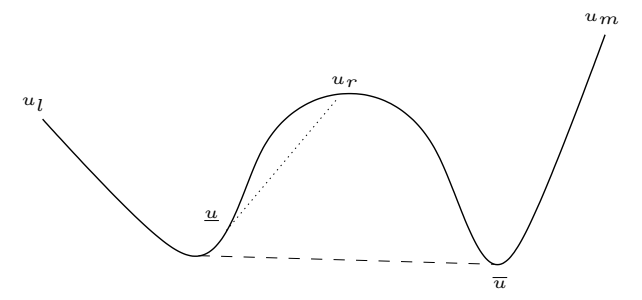

Figure 8: Variation of speed during a non monotone interaction

We introduce (see Figure 8):

$$
\begin{aligned}
& \underline{u}:=\sup \left\{u \in\left[u_{l} ; u_{r}\right) / f(u)=\operatorname{conv}_{\left[u_{l}, u_{r}\right]} f\right\} \\
& \bar{u}:=\inf \left\{u \in\left(u_{r} ; u_{m}\right] / f(u)=\operatorname{conv}_{\left[u_{l}, u_{m}\right]} f\right\} .
\end{aligned}
$$

It is clear that $\operatorname{conv}_{\left[u_{l}, u_{m}\right]} f$ can be obtained by gluing together $\operatorname{conv}_{\left[u_{l}, \bar{u}\right]} f$ and $\operatorname{conv}_{\left[\bar{u} ; u_{m}\right]} f\left(\operatorname{even}\right.$ if $\left.\bar{u}=u_{m}\right)$. It follows that

$$
\bar{\lambda}^{\max }\left(u_{l} ; u_{m}\right) \geq \bar{\lambda}^{\max }\left(u_{l} ; \bar{u}\right)
$$

Moreover it results from the definition of the convex hull that the segment $[(\underline{u}, f(\underline{u})),(\bar{u}, f(\bar{u}))]$ is above the graph of $\operatorname{conv}_{\left[u_{l}, \bar{u}\right]} f$. Hence we have, if $\underline{u}<\bar{u}$,

$$
\bar{\lambda}^{\max }\left(u_{l} ; \bar{u}\right) \geq \frac{f(\bar{u})-f(\underline{u})}{\bar{u}-\underline{u}} .
$$

In the limit case $\underline{u}=\bar{u}\left(=u_{r}\right)$ we have $\bar{\lambda}^{\text {max }}\left(u_{l} ; \bar{u}\right)=f^{\prime}(\bar{u})=f^{\prime}(\underline{u})$. Now it follows from the definition of $\underline{u}$ that $\operatorname{conv}_{\left[u_{l}, u_{r}\right]} f$ is affine in $\left[\underline{u}, u_{r}\right]$ and

$$
\bar{\lambda}^{\max }\left(u_{l} ; u_{r}\right)=\frac{f\left(u_{r}\right)-f(\underline{u})}{u_{r}-\underline{u}},
$$

where the right hand side has to be replaced with $f^{\prime}\left(u_{r}\right)$ in the limit case $\underline{u}=u_{r}$. It follows that

$$
\begin{aligned}
\bar{\lambda}^{\max }\left(u_{l} ; u_{r}\right)-\bar{\lambda}^{\max }\left(u_{l} ; u_{m}\right) & \leq \bar{\lambda}^{\max }\left(u_{l} ; u_{r}\right)-\bar{\lambda}^{\max }\left(u_{l} ; \bar{u}\right) \\
& \leq \frac{f\left(u_{r}\right)-f(\underline{u})}{u_{r}-\underline{u}}-\frac{f(\bar{u})-f(\underline{u})}{\bar{u}-\underline{u}} \\
& \leq \int_{0}^{1}\left[f^{\prime}\left(\underline{u}+\tau\left(u_{r}-\underline{u}\right)\right)-f^{\prime}(\underline{u}+\tau(\bar{u}-\underline{u}))\right] d \tau \\
& \leq \frac{\left\|\left(f^{\prime \prime}\right)_{-}\right\|_{\infty}}{2}\left(\bar{u}-u_{r}\right) \\
& \leq \frac{\left\|\left(f^{\prime \prime}\right)-\right\|_{\infty}}{2}\left(u_{m}-u_{r}\right) .
\end{aligned}
$$

The limit cases are easily included in the above discussion.

- Inequality (42) in the case $u_{m}<u_{l}<u_{r}$. This case results from the general property of the convex hull, symmetrical to (71), that for $a \leq b \leq c$,

$$
\left(\operatorname{conv}_{[a, c]} f\right)^{\prime}(c) \geq\left(\operatorname{conv}_{[b, c]} f\right)^{\prime}(c) \text { for } a \leq b \leq c .
$$

Again we do not need the term $-\left(\left\|\left(f^{\prime \prime}\right)-\right\|_{\infty} / 2\right) \Delta T V$ in the case under view.

- Inequality (41) in the case $u_{m}<u_{l}<u_{r}$. This is the symmetrical case of inequality (42) in the case $u_{l}<u_{r}<u_{m}$. Here we introduce

$$
\begin{array}{r}
\underline{u}:=\inf \left\{u \in\left(u_{l} ; u_{r}\right] / f(u)=\operatorname{conv}_{\left[u_{l}, u_{r}\right]} f\right\}, \\
\bar{u}:=\sup \left\{u \in\left[u_{m} ; u_{l}\right) / f(u)=\operatorname{conv}_{\left[u_{m}, u_{r}\right]} f\right\},
\end{array}
$$

and then it suffices to proceed as for (42) in the case $u_{l}<u_{r}<u_{m}$. 


\subsubsection{Proof of Lemma 4 .}

- Proof of (43). It is a general consequence of the definition of the concave hull (see (71) and (72) for the convex hull), that:

$$
\left(\operatorname{conc}_{[a, c]} f\right)^{\prime}(c) \leq\left(\operatorname{conc}_{[b, c]} f\right)^{\prime}(c) \text { and }\left(\operatorname{conc}_{[a, c]} f\right)^{\prime}(a) \geq\left(\operatorname{conc}_{[a, b]} f\right)^{\prime}(a) \text { for } a \leq b \leq c .
$$

Now consider $u_{m}<u_{r}<u_{l}$; we get

$$
\bar{\lambda}^{\min }\left(u_{l} ; u_{m}\right) \leq \bar{\lambda}^{\min }\left(u_{l} ; u_{r}\right)
$$

Now using that

$$
\bar{\lambda}^{\min }\left(u_{l} ; u_{m}\right) \geq \bar{\lambda}^{\min }\left(u_{m} ; u_{r}\right),
$$

since the fronts $\left(u_{l} ; u_{m}\right)$ and $\left(u_{m} ; u_{r}\right)$ are meeting (and would have met regardless of the additional displacement), we get (43).

- Proof of (46). Consider $u_{r}<u_{l}<u_{m}$. Here (73) yields that

$$
\bar{\lambda}^{\max }\left(u_{l} ; u_{r}\right) \leq \bar{\lambda}^{\max }\left(u_{m} ; u_{r}\right) .
$$

Since again $\left(u_{l} ; u_{m}\right)$ and $\left(u_{m} ; u_{r}\right)$ are meeting, we see that $(74)$ is still valid. Using that for fronts $\alpha$ constructed by our algorithm, $\bar{\lambda}^{\min }(\alpha) \leq \bar{\lambda}^{\max }(\alpha) \leq \bar{\lambda}^{\min }(\alpha)+2 \varepsilon$, we get $(46)$.

- Proof of (44). Consider $u_{m}<u_{r}<u_{l}$. First, from $\operatorname{conv}_{\left[u_{m}, u_{r}\right]} f \leq f$ on $\left[u_{m}, u_{r}\right]$ we immediately deduce that

$$
\bar{\lambda}^{\max }\left(u_{m} ; u_{r}\right)=\left(\operatorname{conv}_{\left[u_{m}, u_{r}\right]} f\right)^{\prime}\left(u_{r}\right) \geq f^{\prime}\left(u_{r}\right) .
$$

(This is the limit case $b \rightarrow c^{-}$in (72)). Now, we distinguish two cases:

- if the interaction point uses the no-splitting strategy: $\tilde{u}=u_{l}$ : then as in (75) we have

$$
\bar{\lambda}^{\min }\left(u_{l} ; u_{r}\right)=\left(\operatorname{conc}_{\left[u_{r}, u_{l}\right]} f\right)^{\prime}\left(u_{l}\right) \leq f^{\prime}\left(u_{l}\right),
$$

and hence

$$
\begin{aligned}
\bar{\lambda}^{\max }\left(u_{l} ; u_{r}\right) & \leq \bar{\lambda}^{\min }\left(u_{l} ; u_{r}\right)+2 \varepsilon \\
& \leq f^{\prime}\left(u_{r}\right)+\left(f^{\prime}\left(u_{l}\right)-f^{\prime}\left(u_{r}\right)\right)+2 \varepsilon \\
& \leq \bar{\lambda}^{\max }\left(u_{m} ; u_{r}\right)+\left(f^{\prime}\left(u_{l}\right)-f^{\prime}\left(u_{r}\right)\right)_{+}+2 \varepsilon .
\end{aligned}
$$

- if the interaction point uses the splitting strategy: then it follows from the construction of the approximate solver that

$$
\bar{\lambda}^{\min }\left(\tilde{u} ; u_{r}\right)=f^{\prime}(\tilde{u})=\bar{\lambda}^{\max }\left(u_{l} ; u_{r}\right)-\varepsilon,
$$

and hence

$$
\begin{aligned}
\bar{\lambda}^{\max }\left(u_{l} ; u_{r}\right) & =f^{\prime}\left(u_{r}\right)+\left(f^{\prime}(\tilde{u})-f^{\prime}\left(u_{r}\right)\right)+\varepsilon \\
& \leq \bar{\lambda}^{\max }\left(u_{m} ; u_{r}\right)+\left(f^{\prime}(\tilde{u})-f^{\prime}\left(u_{r}\right)\right)_{+}+\varepsilon .
\end{aligned}
$$

- Proof of (45). Consider $u_{r}<u_{l}<u_{m}$. Here we start from

$$
\bar{\lambda}^{\min }\left(u_{l} ; u_{m}\right)=\left(\operatorname{conv}_{\left[u_{l}, u_{m}\right]} f\right)^{\prime}\left(u_{l}\right) \leq f^{\prime}\left(u_{l}\right) .
$$

Again, we distinguish the two cases:

- if the interaction point uses the no-splitting strategy: $\tilde{u}=u_{r}$ : then

$$
\bar{\lambda}^{\max }\left(u_{l} ; u_{r}\right)=\left(\operatorname{conc}_{\left[u_{r}, u_{l}\right]} f\right)^{\prime}\left(u_{r}\right) \geq f^{\prime}\left(u_{r}\right),
$$

and hence

$$
\begin{aligned}
\bar{\lambda}^{\min }\left(u_{l} ; u_{r}\right) & \geq \bar{\lambda}^{\max }\left(u_{l} ; u_{r}\right)-2 \varepsilon \\
& \geq f^{\prime}\left(u_{l}\right)-\left(f^{\prime}\left(u_{l}\right)-f^{\prime}\left(u_{r}\right)\right)-2 \varepsilon \\
& \geq \bar{\lambda}^{\text {min }}\left(u_{l} ; u_{m}\right)-\left(f^{\prime}\left(u_{l}\right)-f^{\prime}\left(u_{r}\right)\right)_{+}-2 \varepsilon .
\end{aligned}
$$

- if the interaction point uses the splitting strategy: then it follows again that

$$
\bar{\lambda}^{\max }\left(u_{l}, \tilde{u}\right)=f^{\prime}(\tilde{u})=\bar{\lambda}^{\min }\left(u_{l} ; u_{r}\right)+\varepsilon,
$$

and hence

$$
\begin{aligned}
\bar{\lambda}^{\min }\left(u_{l} ; u_{r}\right) & =f^{\prime}\left(u_{l}\right)+\left(f^{\prime}(\tilde{u})-f^{\prime}\left(u_{l}\right)\right)-\varepsilon \\
& \geq \bar{\lambda}^{\text {min }}\left(u_{l} ; u_{m}\right)-\left(f^{\prime}\left(u_{l}\right)-f^{\prime}(\tilde{u})\right)_{+}-\varepsilon .
\end{aligned}
$$




\subsection{Proof of the interaction estimates on the inner speed variation.}

Note that a version of Proposition 4 was proved in [8] in the case of systems: in this case there is an additional interaction term in the right hand side of (26). Here we prove that we can get rid of the additional term in (26) and that we can take $C_{i}$ as in (29) for (27)-(28). These inequalities are rather direct, except in the case of (27)-(28) for a negative outgoing wave (i.e. $u_{r}<u_{l}$ ).

\subsubsection{Proof of (26).}

Let us briefly explain (26). We only treat the case $u_{l}<u_{m}<u_{r}$ since the other one is similar. We introduce

$$
\begin{gathered}
u_{1}:=\max \left\{u \in\left[u_{l}, u_{m}\right], f(u)=\operatorname{conv}_{\left[u_{l}, u_{r}\right]} f(u)\right\}, \\
u_{2}:=\min \left\{u \in\left[u_{m}, u_{r}\right], f(u)=\operatorname{conv}_{\left[u_{l}, u_{r}\right]} f(u)\right\} .
\end{gathered}
$$

- Suppose that $u_{l}<u_{1} \leq u_{2}<u_{r}$. Then $\operatorname{conv}_{\left[u_{l}, u_{r}\right]} f$ coincides with $\operatorname{conv}_{\left[u_{l}, u_{m}\right]} f\left(\right.$ resp. with $\left.\operatorname{conv}_{\left[u_{m}, u_{r}\right]} f\right)$ on $\left[u_{l}, u_{1}\right]$ (resp. on $\left.\left[u_{2}, u_{r}\right]\right)$, hence

$$
\begin{aligned}
\vartheta\left(u_{l} ; u_{r}\right) & =\bar{\lambda}^{\max }\left(u_{m} ; u_{r}\right)-\bar{\lambda}^{\min }\left(u_{l} ; u_{m}\right) \\
& =\bar{\lambda}^{\max }\left(u_{m} ; u_{r}\right)-\bar{\lambda}^{\min }\left(u_{m} ; u_{r}\right)+\bar{\lambda}^{\min }\left(u_{m} ; u_{r}\right)-\bar{\lambda}^{\min }\left(u_{l} ; u_{m}\right) \\
& \leq \vartheta\left(u_{m} ; u_{r}\right)+\left(\bar{\lambda}^{\min }\left(u_{m} ; u_{r}\right)-\bar{\lambda}^{\min }\left(u_{l} ; u_{m}\right)\right)_{+} .
\end{aligned}
$$

- Suppose $u_{l}=u_{1}<u_{2}<u_{r}$ (resp. $\left.u_{l}<u_{1}<u_{2}=u_{r}\right)$. In that case, we see that $\vartheta\left(u_{l} ; u_{r}\right)=\vartheta\left(u_{2}, u_{r}\right)$ (resp. $\left.\vartheta\left(u_{l} ; u_{r}\right)=\vartheta\left(u_{l}, u_{1}\right)\right)$ and the conclusion follows.

- The case $u_{l}=u_{1}<u_{2}=u_{r}$ involves $\vartheta\left(u_{l} ; u_{r}\right)=0$.

\subsubsection{Proof of (27)-(28): the case of a positive outgoing wave.}

The cases of positive outgoing waves $\left(u_{l}<u_{r}\right)$ follow directly from Lemma 3. Moreover, we can obtain the result with a better constant than (29). As a matter of fact, if we subtract (41) from (42) we already get the result with constant $\left\|\left(f^{\prime \prime}\right)_{-}\right\|_{\infty} \leq \sqrt{\left\|\left(f^{\prime \prime}\right)_{-}\right\|_{\infty}\left\|f^{\prime \prime}\right\|_{\infty}}$; but taking a closer look, we see that we can obtain $\left\|\left(f^{\prime \prime}\right)_{-}\right\|_{\infty} / 2$ as a constant by using

- (42) and (71) in the case $u_{l}<u_{r}<u_{m}$,

- (41) and (72) in the case $u_{m}<u_{l}<u_{r}$.

As we will see, the two cases where $u_{l}>u_{r}$ need a different treatment.

\subsubsection{Proof of (27)-(28): the case of a negative outgoing wave.}

It is rather clear that if we used the same strategy as is the previous paragraph in the case of a negative outgoing wave $\left(u_{l}>u_{r}\right)$, the error terms would be of the type $\left\|\left(f^{\prime \prime}\right)_{+}\right\|_{\infty} \Delta T V$.

We restrict our attention to inequality (28) in the case $u_{r}<u_{l}<u_{m}$ since inequality (27) in the case $u_{m}<u_{r}<u_{l}$ is a symmetrical situation. The proof of (28) follows several steps.

1. Reduction to a non degenerate flux. It will be convenient to work with a non-degenerate flux, as it is defined in [8]: in the scalar case, a non-degenerate flux is a flux $f \in C^{\infty}(\mathbb{R} ; \mathbb{R})$ satisfying:

$$
\left\{u \in \mathbb{R} / f^{\prime \prime}(u)=f^{\prime \prime \prime}(u)=0\right\}=\emptyset .
$$

The basic point is the following.

Lemma 7. The set of non-degenerate fluxes is a Baire second category set, for Whitney's topology.

Lemma 7 is a (very) particular case of [8, Theorem 2.3]. Also, it could be proved elementarily by using Baire's Theorem.

Using Lemma 7, one sees that it is sufficient to prove (28) in the case of a non-degenerate flux. Indeed, one can find a sequence $\left(f^{n}\right)$ of non-degenerate fluxes, converging to $f$ for the $C^{k}$ norm on any compact subset of $\mathbb{R}$ (in particular on $\left.\left[-\left\|u_{0}\right\|_{\infty},\left\|u_{0}\right\|_{\infty}\right]\right)$. It is then enough to pass to the limit in

$$
\begin{array}{r}
{\left[\left(\operatorname{conc}_{\left[u_{r}, u_{l}\right]} f_{n}\right)^{\prime}\left(u_{r}\right)-\left(\operatorname{conc}_{\left[u_{r}, u_{l}\right]} f_{n}\right)^{\prime}\left(u_{l}\right)\right]-\left[\left(\operatorname{conc}_{\left[u_{r}, u_{m}\right]} f_{n}\right)^{\prime}\left(u_{r}\right)-\left(\operatorname{conc}_{\left[u_{r}, u_{m}\right]} f_{n}\right)^{\prime}\left(u_{m}\right)\right]} \\
\leq \sqrt{\left\|\left(f_{n}^{\prime \prime}\right)-\right\|_{\infty}\left\|f_{n}^{\prime \prime}\right\|_{\infty}}\left(u_{m}-u_{l}\right) .
\end{array}
$$


The passage to the limit comes for instance from:

$$
\left\{\begin{array}{l}
\left|\left(\operatorname{conc}_{[a, b]} f\right)^{\prime}(a)-\left(\operatorname{conc}_{[a, b]} g\right)^{\prime}(a)\right| \leq\left\|f^{\prime}-g^{\prime}\right\|_{\infty} \\
\left|\left(\operatorname{conc}_{[a, b]} f\right)^{\prime}(b)-\left(\operatorname{conc}_{[a, b]} g\right)^{\prime}(b)\right| \leq\left\|f^{\prime}-g^{\prime}\right\|_{\infty}
\end{array}\right.
$$

Let us explain the first inequality. This is easily adapted for the second one and in the case of the convex hull too. It is clear from the definition that

$$
\left(\operatorname{conc}_{[a, b]} f\right)^{\prime}(a)=\inf \{\lambda \in \mathbb{R} / f(x) \leq f(a)+\lambda(x-a) \text { on }[a, b]\} .
$$

One can assume $f(a)=g(a)$ since constants do not intervene here. Now (76) follows from

$$
\begin{aligned}
\left\{\lambda+\left\|f^{\prime}-g^{\prime}\right\|_{\infty} \in \mathbb{R} / g(x) \leq g(a)+\lambda(x-a) \text { on }[a, b]\right\} & \\
& \subset\{\lambda \in \mathbb{R} / f(x) \leq f(a)+\lambda(x-a) \text { on }[a, b]\} .
\end{aligned}
$$

2. Second reduction. Now let us prove that we can reduce to the case where:

$$
\forall u \in\left[u_{l}, u_{m}\right), \quad \operatorname{conc}_{\left[u_{r}, u\right]} f \text { is not affine. }
$$

Indeed:

- if $\operatorname{conc}_{\left[u_{r}, u_{l}\right]} f$ is affine then the result is trivial,

- else, if $\operatorname{conc}_{\left[u_{r}, u\right]} f$ is affine for some $u \in\left(u_{l}, u_{m}\right)$ (but not for $u=u_{l}$ ), then we introduce

$$
\underline{u}:=\min \left\{u \in\left(u_{l}, u_{m}\right) / \operatorname{conc}_{\left[u_{r}, u\right]} f \text { is affine }\right\}>u_{l} .
$$

Then replacing $u_{m}$ with $\underline{u}$ makes the new statement (28) to prove stronger (the left hand side is unchanged, and the right hand side is diminished, since $\vartheta\left(u_{r} ; \underline{u}\right)=0$, and the term $\Delta T V:=u_{m}-u_{l}$ is also smaller), and now we are in the situation described by (77).

From now on we suppose that the flux is non-degenerate and that (77) holds.

3. Proof in the reduced case. Define $L:\left[u_{l}, u_{m}\right] \ni u \mapsto\left(\operatorname{conc}_{\left[u_{r}, u\right]} f\right)^{\prime}(u)$. Our goal is to prove that $L$ is continuous and right-derivable in $\left[u_{l}, u_{m}\right]$, with its derivative satisfying

$$
\frac{d}{d u^{+}} L(u) \leq \sqrt{\left\|\left(f^{\prime \prime}\right)_{-}\right\|_{\infty}\left\|f^{\prime \prime}\right\|_{\infty}}
$$

(Recall that $u_{r}<u_{l}<u_{m}$ ) For this purpose, we pick $u \in\left[u_{l}, u_{m}\right]$, and discuss according to the situation at the point $u$.

- If $u$ is a "standard" contact point, in the sense that:

$$
\left(\operatorname{conc}_{\left[u_{r}, u\right]} f\right)^{\prime}(u)=f^{\prime}(u) \text { and } f^{\prime \prime}(u)<0 .
$$

Then in a right neighborhood of $u$, one constructs $\operatorname{conc}_{\left[u_{r}, \cdot\right]} f$ by extending $\operatorname{conc}_{\left[u_{r}, u\right]} f$ with $f$. Hence $L$ is continuous and derivable in such a neighborhood, and moreover it satisfies (78) (even, with 0 on the right hand side).

- If $u$ is a "critical" contact point, in the sense that:

$$
\left(\operatorname{conc}_{\left[u_{r}, u\right]} f\right)^{\prime}(u)=f^{\prime}(u) \text { and } f^{\prime \prime}(u)=0 .
$$

At such a point, one has $f^{\prime \prime \prime}(u) \neq 0$. As a concave hull of a function is above this function and below its tangents, it follows that $f^{\prime \prime \prime}(u)>0$. One can locally define the application

$$
\begin{aligned}
G(v, w) & :=\left\{\begin{array}{l}
\frac{1}{w-v}\left(\frac{f(w)-f(v)}{w-v}-f^{\prime}(v)\right) \text { for } w \neq v, \\
\frac{1}{2} f^{\prime \prime}(v) \text { otherwise }
\end{array}\right. \\
& =\int_{0}^{1}(1-\theta) f^{\prime \prime}(v+\theta(w-v)) d \theta .
\end{aligned}
$$

(This is the standard way to define the mixed curve for systems, see e.g. [10, Theorem 5.1].) Clearly, $G$ is regular, and moreover

$$
\partial_{v} G(u, u)=\frac{1}{3} f^{\prime \prime \prime}(u)>0 .
$$


Hence by the implicit function theorem, one can locally define around $u$ a regular application $\varphi$ solution to

$$
\frac{f(w)-f(\varphi(w))}{w-\varphi(w)}=f^{\prime}(\varphi(w))
$$

and satisfying $\varphi(u)=u$. Note that $\partial_{w} G(u, u)=\frac{1}{6} f^{\prime \prime \prime}(u)>0$, hence locally $\varphi$ is decreasing. Consequently, for $w$ in a right neighborhood of $u$, one constructs $\operatorname{conc}_{\left[u_{r}, w\right]} f$ by gluing together $\operatorname{conc}_{\left[u_{r}, \varphi(w)\right]} f$ and the affine function connecting the points $(\varphi(w), f(\varphi(w)))$ and $(w, f(w))$.

It follows that in such a neighborhood,

$$
L(w)=f^{\prime}(\varphi(w))
$$

Now differentiating (79) with respect to $w$ yields

$$
\frac{d}{d w} f^{\prime}(\varphi(w))=\frac{f^{\prime}(w)-f^{\prime}(\varphi(w))}{w-\varphi(w)}
$$

To estimate the right-hand side, we temporarily admit the following lemma.

Lemma 8. Let $a<b$ and $g \in C^{0}([a, b] ; \mathbb{R})$ such that

$$
\int_{a}^{b}(b-x) g(x) d x=0
$$

Then

$$
\frac{1}{b-a} \int_{a}^{b} g \leq \sqrt{\left\|g_{-}\right\|_{\infty}\left(\left\|g_{-}\right\|_{\infty}+\left\|g_{+}\right\|_{\infty}\right)}-\left\|g_{-}\right\|_{\infty} \leq \sqrt{\left\|g_{-}\right\|_{\infty}\left\|g_{+}\right\|_{\infty}}
$$

Now (79) and Taylor's integral formula involve that (81) is satisfied with

$$
g=f^{\prime \prime}, \quad a=\varphi(w) \text { and } b=w .
$$

Applying (82) yields, together with (80), that (78) is satisfied.

- If $u$ is a "transversal" touching point, in the sense that:

$$
\left(\operatorname{conc}_{\left[u_{r}, u\right]} f\right)^{\prime}(u)<f^{\prime}(u) .
$$

Then we introduce $\underline{u}$ as

$$
\underline{u}:=\inf \left\{v \in\left[u_{r}, u\right) /\left(\operatorname{conc}_{\left[u_{r}, u\right]} f\right)(v)=f(v) \text { and }\left(\operatorname{conc}_{\left[u_{r}, u\right]} f\right)^{\prime}(v)=\left(\operatorname{conc}_{\left[u_{r}, u\right]} f\right)^{\prime}(u)\right\} .
$$

The case $\underline{u}=u_{r}$ is excluded by condition (77). Hence we deduce

$$
\frac{f(\underline{u})-f(u)}{\underline{u}-u}=f^{\prime}(\underline{u})
$$

Moreover, for $v<\underline{u}$ we have $\left(\operatorname{conc}_{\left[u_{r}, u\right]} f\right)^{\prime}(v)>\left(\operatorname{conc}_{\left[u_{r}, u\right]} f\right)^{\prime}(u)$, hence it follows, together with the nondegeneracy of $f$, that $\operatorname{conc}_{\left[u_{r}, u\right]} f$ coincides with $f$ in some left neighborhood of $\underline{u}$. Note that one cannot have $f^{\prime \prime}(\underline{u})=0$, because the tangent to $f$ at $\underline{u}$ is above the graph of conc ${ }_{\left[u_{r}, u\right]} f$, hence of the one of $f$ (and the flux is non-degenerate). Hence $f^{\prime \prime}(\underline{u})<0$. It follows that one can define a regular function $\varphi$ in a right neighborhood of $u$, in such a way that $\varphi(u)=\underline{u}$ and (79) applies. It is indeed sufficient to apply implicit functions theorem to

$$
H(v, w):=\frac{f(w)-f(v)}{w-v}-f^{\prime}(v),
$$

which we can do because as a consequence of (83), one has

$$
\partial_{v} H(\underline{u}, u):=-f^{\prime \prime}(\underline{u})>0 .
$$

Now as previously one constructs $\operatorname{conc}_{\left[u_{r}, w\right]} f$ for $w$ in a small right neighborhood of $u$, by gluing together $\operatorname{conc}_{\left[u_{r}, \varphi(w)\right]} f$ and the affine function connecting the points $(\varphi(w), f(\varphi(w)))$ and $(w, f(w))$. Again we apply Lemma 8, and (78) follows.

To conclude, we have (78) in all (interesting) cases, and it follows that

$$
\bar{\lambda}^{\text {min }}\left(u_{l} ; u_{r}\right) \geq \bar{\lambda}^{\min }\left(u_{m} ; u_{r}\right)-\sqrt{\left\|\left(f^{\prime \prime}\right)_{-}\right\|_{\infty}\left\|f^{\prime \prime}\right\|_{\infty}}\left(u_{m}-u_{l}\right) .
$$

Since on another side

$$
\bar{\lambda}^{\max }\left(u_{l} ; u_{r}\right) \leq \bar{\lambda}^{\max }\left(u_{m} ; u_{r}\right),
$$

as seen from (73), we obtain (28). 
Remark 8. Observe that (84) is not in contradiction with Remark 4, since it is only valid when (77) is satisfied. In a situation where $\operatorname{conc}_{\left[u_{r}, u\right]} f$ is affine for $u$ in some interval $I$, the inner speed variation $\vartheta\left(u_{r} ; u\right)$ is null for all $u \in I$, while $\bar{\lambda}^{\max }\left(u_{r} ; u\right)=\bar{\lambda}^{\min }\left(u_{r} ; u\right)$ may vary, even if $f^{\prime \prime} \geq 0$.

Proof of Lemma 8. Of course, we may exclude the trivial case $g=0$ from the discussion. We introduce some parameter $\mu \in[0, b-a]$; then we have the following inequalities:

$$
\begin{aligned}
\int_{a}^{b} g(x) d x & \leq \int_{a}^{b-\mu} \frac{b-x}{\mu} g_{+}(x) d x+\int_{b-\mu}^{b} g_{+}(x) d x-\int_{a}^{b} g_{-}(x) d x \\
& \leq \int_{a}^{b} \frac{b-x}{\mu} g_{+}(x) d x+\int_{b-\mu}^{b}\left(1-\frac{b-x}{\mu}\right) g_{+}(x) d x-\int_{a}^{b} g_{-}(x) d x \\
& =\int_{a}^{b}\left(\frac{b-x}{\mu}-1\right) g_{-}(x) d x+\int_{b-\mu}^{b}\left(1-\frac{b-x}{\mu}\right) g_{+}(x) d x \\
& \leq\left\|g_{-}\right\| \int_{a}^{b-\mu}\left(\frac{b-x}{\mu}-1\right) d x+\left\|g_{+}\right\|_{\infty} \int_{b-\mu}^{b}\left(1-\frac{b-x}{\mu}\right) d x \\
& =\left[\frac{(b-a)^{2}}{2 \mu}+\frac{\mu}{2}-(b-a)\right]\left\|g_{-}\right\|_{\infty}+\frac{\mu}{2}\left\|g_{+}\right\|_{\infty} .
\end{aligned}
$$

Taking

$$
\mu:=(b-a) \sqrt{\frac{\left\|g_{-}\right\|_{\infty}}{\left\|g_{-}\right\|_{\infty}+\left\|g_{+}\right\|_{\infty}}}(\in[0, b-a])
$$

yields the first inequality. The second one is just the subadditivity of the square root.

Remark 9. Clearly, one has also

$$
\sqrt{\left\|g_{-}\right\|_{\infty}\left(\left\|g_{-}\right\|_{\infty}+\left\|g_{+}\right\|_{\infty}\right)}-\left\|g_{-}\right\|_{\infty} \leq \frac{\left\|g_{+}\right\|_{\infty}}{2} .
$$

But one cannot compare in general $\sqrt{\left\|g_{-}\right\|_{\infty}\left(\left\|g_{-}\right\|_{\infty}+\left\|g_{+}\right\|_{\infty}\right)}-\left\|g_{-}\right\|_{\infty}$ and $\left\|g_{-}\right\|_{\infty} / 2$. This explains the maxima appearing in formula (14), see Paragraph 6.4.

\subsection{Proofs of Lemmas 5 and 6.}

\subsubsection{Proof of Lemma 5}

That the selection process is transitive is a straightforward consequence of the construction. Now let us establish the second assertion. Consider two fronts $\alpha$ and $\beta$ and a front $\gamma \in \mathcal{T}(\alpha) \cap \mathcal{T}(\beta)$. Of course one can suppose $\alpha \neq \beta$. Starting from $\gamma$, move up in the tree. As the algorithm of Paragraph 3.3 stops at any interaction point using the splitting strategy, at each interaction point which we meet in the process, there is only one outgoing front. Hence as long as in this process we do not have met $\alpha$ or $\beta$, the front that we consider is necessarily in $\mathcal{T}(\alpha) \cap \mathcal{T}(\beta)$. When we reach $\alpha$ or $\beta$, the transitivity yields the conclusion.

Now, let us establish (47). Given a front $\gamma$, we call $t_{\gamma}$ its time of generation, given an interaction point $P$, we call $t_{P}$ its time, i.e. its first projection. We consider a front $\alpha$. We exclude the case $\vartheta_{\alpha} \leq \varepsilon$ from the discussion since (47) is trivial in that case (in particular this excludes the case $t_{\alpha}=0$ ). Call $\tilde{t}$ the minimal time of a leaf of the tree $\mathcal{T}(\alpha)$. We prove (85) by induction, precisely, we prove that for any $s \in[\tilde{t}, t]$,

$$
\max \left\{\vartheta_{\beta}, \beta \in \mathcal{T}(\alpha) \text { such that } t_{\beta}<s\right\}-\varepsilon \leq \sqrt{\left\|\left(f^{\prime \prime}\right)_{-}\right\|_{\infty}\left\|f^{\prime \prime}\right\|_{\infty}} \sum_{\substack{P \in \mathcal{V}(\alpha), \\ \text { s. t. } t_{p}<s}}[\Delta T V](P) .
$$

We start at the minimal time $\tilde{t}$. Considering the algorithm of Paragraph 3.3, at the corresponding interaction point:

- or it is an interaction using the splitting strategy: this is excluded, since from such an interaction point, outgoes only fronts with inner speed variation no more than $\varepsilon$; we have excluded this case.

- or it is a monotone interaction (using the no-splitting strategy): in that case, both incoming fronts had an inner speed variation not more than $\varepsilon$, while the outgoing front has an inner speed variation larger than $\varepsilon$. Using (26) and remarking that meeting of the incoming fronts involves that the last term in (26) vanishes, we conclude that this case is also excluded. 
- or it is a non-monotone interaction using the no-splitting strategy: in that case, the larger incoming front (the one with the same sign as the outgoing one) has an inner wave speed no more than $\varepsilon$, while the outgoing one has inner wave speed more than $\varepsilon$. Using (27)-(28), we see that this involves that (85) is valid for times immediately after $\tilde{t}$.

After the initial time $\tilde{t}$, both sides of (85) can only change at interaction times. Consider an interaction time $\bar{t}$ which modifies a side of (85). There are two possibilities for the right hand side to change:

- or the interaction point considered is not an inner vertex of $\mathcal{T}(\alpha)$, that is, none of the incoming fronts belongs to the tree: in that case, the previous discussion for time $\tilde{t}$ can be reproduced.

- or the interaction point considered is an inner vertex of $\mathcal{T}(\alpha)$, that is at least one of the incoming fronts at this point was already in $\mathcal{T}(\alpha)$. In that case, considering the algorithm of Paragraph 3.3, there are only two possibilities:

- or the interaction uses the no-splitting strategy and is monotone: in that case, the conclusion comes from (26) and from the "induction hypothesis" (the fact that (85) was valid for previous times),

- or the interaction uses the no-splitting strategy and is non-monotone, and the conclusion comes from the induction hypothesis and (27)-(28).

This concludes the proof of (85) and hence of Lemma 5.

Remark 10. One could have defined the isv source tree differently, for instance by picking only the front with the larger inner speed variation at a monotone interaction point where both incoming fronts have an inner speed variation larger than $\varepsilon$. Lemma 5 would still be valid in that case.

\subsubsection{Proof of Lemma 6}

The transitivity is again a direct consequence of the construction. Concerning the second assertion: again, we consider $\alpha$ and $\beta$ two fronts such that $\widehat{\mathcal{T}}(\alpha) \cap \widehat{\mathcal{T}}(\beta) \neq \emptyset$ and moreover we assume $\alpha \neq \beta$. Again, one can go up (forward in time) in the tree without ambiguity, and the fronts considered stay in both trees, until we meet $\alpha$ or $\beta$. The transitivity yields the conclusion.

Now, we aim at establishing (48). We use the same notations as in the previous paragraph, and we perform an induction on the size of the tree (the number of its fronts). We call $\tilde{t}$ the minimal time of a leaf of the tree $\hat{\mathcal{T}}(\alpha)$; here $\tilde{t}>0$ because the tree under view is good in the sense of Paragraph 3.4 (the outer speed variation source tree can reach $t=0$ in general). We exclude from the discussion the cases where $\alpha$ is positive (by assumption) or has a nonpositive outer speed variation (by triviality). Now:

- if the tree has only one front, then (48) is a trivial consequence of (36),

- next, we assume that we have proved (48) for any good outer speed variation source tree which is composed of no more than $n$ fronts. Let us show that this is valid for trees with $n+1$ fronts. Suppose that $\widehat{\mathcal{T}}(\alpha)$ is such a tree $(n \geq 1)$. We go back in time starting from $\alpha$. The first interaction point that we find is necessarily a monotone interaction point (otherwise, there is only one front in this tree). There are two possibilities:

- or both incoming fronts have a positive outer speed variation,

- or only one of the incoming fronts has a positive outer speed variation,

In both cases we can apply the induction hypothesis on each tree of the incoming fronts that have a positive outer speed variation, and then the conclusion simply comes from

$$
f^{\prime}\left(u_{r}\right)-f^{\prime}\left(u_{l}\right)=f^{\prime}\left(u_{r}\right)-f^{\prime}\left(u_{m}\right)+f^{\prime}\left(u_{m}\right)-f^{\prime}\left(u_{l}\right) .
$$

This concludes the proof of (85) and hence of Lemma 6 .

\section{Complements.}

\subsection{Replacing $\sqrt{\left\|\left(f^{\prime \prime}\right)_{-}\right\|_{\infty}\left\|f^{\prime \prime}\right\|_{\infty}}$ with $\sqrt{\left\|\left(f^{\prime \prime}\right)_{+}\right\|_{\infty}\left\|f^{\prime \prime}\right\|_{\infty}}$}

A first obvious remark is that we can readily infer Proposition 2 from Proposition 1 by noting that $u$ is an entropy solution of (1) if and only if $v:=-u$ is an entropy solution of

$$
v_{t}+(g(v))_{x}=0 \text { for }(t, x) \in \mathbb{R}^{+} \times \mathbb{R},
$$


where

$$
g(\cdot):=-f(-\cdot)
$$

(Think of the vanishing viscosity approach for instance.) Hence applying Proposition 1 on $v$ gives Proposition 2 on $u$.

Let us underline that doing so, we somehow estimate parts of $u$ which are different from those that we consider when proving Proposition 1. Indeed, when doing as described above, we invert the respective roles of positive and negative fronts. In particular, if we had wished to prove Proposition 2 directly, in Section 4 we would have inverted the roles of positive and negative fronts, that is, negative fronts would have been continued as front paths, while positive fronts would have been continued as trees, the abandoned fronts would have been positive ones, etc.

Remark in particular that Proposition 4 is valid when instead of taking $C_{i}=\sqrt{\left\|\left(f^{\prime \prime}\right)_{-}\right\|_{\infty}\left\|f^{\prime \prime}\right\|_{\infty}}$, we take $C_{i}=\sqrt{\left\|\left(f^{\prime \prime}\right)_{+}\right\|_{\infty}\left\|f^{\prime \prime}\right\|_{\infty}}$ (in that case, the proofs of Paragraphs 5.2.2 and 5.2.3 are inverted). Note also that the equivalent of Lemma 3 for negative fronts naturally yields a coefficient $\left\|\left(f^{\prime \prime}\right)_{+}\right\|_{\infty}$ instead of $\left\|\left(f^{\prime \prime}\right)_{-}\right\|_{\infty}$, and that an equivalent of Lemma 4 can be proved for positive fronts.

\subsection{Replacing $\sqrt{\left\|\left(f^{\prime \prime}\right)_{-}\right\|_{\infty}\left\|\left(f^{\prime \prime}\right)_{+}\right\|_{\infty}}$ with $\left\|f^{\prime \prime}\right\|_{\infty} / 2$}

In this paragraph, we wish to underline that the proof would have been way shorter and simpler if the goal had been to prove (15) instead of (11).

We list the simplifications of that case:

- The proof of Proposition 4 with $C_{i}=\|f\|_{\infty} / 2$ is simpler since the analysis of Paragraph 5.2.2 is sufficient in that case; hence Paragraph 5.2.3 is unnecessary;

- Lemma 3 is valid for both positive and negative fronts when one allows a coefficient $\left\|f^{\prime \prime}\right\|_{\infty} / 2$ instead of $\left\|\left(f^{\prime \prime}\right)_{-}\right\|_{\infty} / 2$.

- The most important consequence of that is the following one: since Lemma 3 is always valid, we do not need to distinguish between positive and negative fronts, and they all can be treated as we treat positive fronts in Paragraph 4.2.1. Hence no trees are in order here: we do not need Lemmas 5 and 6 , nor the algorithms of Paragraphs 4.2.2 and 4.2.3; in the proof of Proposition 5, only the reasoning for positive front is used (in particular we need not mark interaction points); finally, Paragraph 4.4.3 is unnecessary.

\subsection{Proof of Theorem $1^{\prime}$}

Fix $I:=[a, b]$. In order to establish Theorem $1^{\prime}$, it is sufficient to prove, instead of (11):

$$
\sum_{i=1}^{n} f^{\prime}\left(u\left(T, y_{i}\right)\right)-f^{\prime}\left(u\left(T, x_{i}\right)\right) \leq \frac{b-a}{T}+\sqrt{\left\|\left(f^{\prime \prime}\right)_{-}\right\|_{\infty}\left\|f^{\prime \prime}\right\|_{\infty}}[T V(u(0, \cdot))-T V(u(T, \cdot))],
$$

for any family of $2 n$ real numbers $x_{i}$ and $y_{i}$ satisfying

$$
a \leq x_{1} \leq y_{1} \leq \cdots \leq x_{n} \leq y_{n} \leq b .
$$

Considering the supremum over all such families $\left(x_{i}, y_{i}\right)$ in (86) yields (13). Now in order to prove (86), it is enough to establish, instead of (49):

$$
\sum_{i=1}^{n} f^{\prime}\left(u_{\varepsilon_{\nu}}\left(T, y_{i}\right)\right)-f^{\prime}\left(u_{\varepsilon_{\nu}}\left(T, x_{i}\right)\right) \leq \frac{b-a}{T}+\sqrt{\left\|\left(f^{\prime \prime}\right)_{-}\right\|_{\infty}\left\|f^{\prime \prime}\right\|_{\infty}}\left[T V\left(u_{\varepsilon_{\nu}}(0, \cdot)\right)-T V\left(u_{\varepsilon_{\nu}}(T, \cdot)\right)\right]+\mathcal{O}\left(\varepsilon_{\nu}\right),
$$

where of course the term $\mathcal{O}\left(\varepsilon_{\nu}\right)$ can depend on $n$.

Now we perform the same analysis as in the proof of Proposition 1 for each couple $\left(x_{i}, y_{i}\right)$ : for each $i$ we determine front paths $\gamma_{i}^{x}$ and $\gamma_{i}^{y}$, their meeting time $\tilde{t}_{i}$ when going back in time (set to be 0 if they do not meet), and sets $\mathcal{D}\left(\bar{\gamma}_{i}^{x}\right), \mathcal{D}\left(\bar{\gamma}_{i}^{y}\right)$ and $\mathcal{D}(\alpha)$ for abandoned trees. If during the process of Paragraph 4.2.3 we have abandoned all the fronts between $x_{i}$ and $y_{i}$, we consider that $\mathcal{D}\left(\bar{\gamma}_{i}^{x}\right)=\mathcal{D}\left(\bar{\gamma}_{i}^{y}\right)=\emptyset$ (see Paragraph 4.4.3). Now there are two cases in order.

First case. Let us assume that, for all $1 \leq i<j \leq n$, the front paths $\gamma_{i}^{y}$ and $\gamma_{j}^{x}$ do not meet during times $t>\max \left(\tilde{t}_{i}, \tilde{t}_{j}\right)$.

1. Let us show that all the sets $\mathcal{D}\left(\bar{\gamma}_{i}^{x}\right), \mathcal{D}\left(\bar{\gamma}_{i}^{y}\right)$ and $\mathcal{D}(\alpha)$ for abandoned trees are disjoint. This can be seen as follows: 
- The points in $\mathcal{D}\left(\bar{\gamma}_{i}^{x}\right)$ and $\mathcal{D}\left(\bar{\gamma}_{j}^{y}\right)$ with negative outgoing fronts and the points in $\mathcal{D}(\alpha)$ for abandoned fronts can be shown to be distinct as in Paragraph 4.3.7: "marked interaction points" with negative outgoing fronts in a set $\mathcal{D}$ can be connected in a unique way to front $\alpha$ at time $T$.

- Concerning the points in $\mathcal{D}\left(\bar{\gamma}_{i}^{x}\right)$ and $\mathcal{D}\left(\bar{\gamma}_{j}^{y}\right)$ with positive outgoing fronts, we remark that the various paths $\left(\gamma_{i}^{x}\right)_{\mid t>\tilde{t}_{i}}$ and $\left(\gamma_{j}^{y}\right)_{\mid t>\tilde{t}_{j}}$ have no intersection:

- two front paths $\gamma_{i}^{x}$ and $\gamma_{i}^{y}$ cannot intersect at a time $t>\tilde{t}_{i}$ by the definition of $\tilde{t}_{i}$;

- two front paths $\gamma_{i}^{y}$ and $\gamma_{j}^{x}$ with $i<j$ cannot intersect at a time $t>\max \left(\tilde{t}_{i}, \tilde{t}_{j}\right)$ : this is precisely our assumption of the "first case",

- two front paths $\gamma_{i}^{y}$ and $\gamma_{j}^{x}$ with $i>j$ cannot intersect at a time $t>\max \left(\tilde{t}_{i}, \tilde{t}_{j}\right)$, because in that case $\gamma_{j}^{y}$ and $\gamma_{i}^{x}$ are between $\gamma_{j}^{x}$ and $\gamma_{i}^{y}$, and cannot intersect themselves nor exit from the zone delimited by $\gamma_{j}^{x}$ and $\gamma_{i}^{y}$.

2. For each $i$, the analysis of Section 4 shows that

$$
f^{\prime}\left(u_{\varepsilon_{\nu}}\left(t, y_{i}\right)\right)-f^{\prime}\left(u_{\varepsilon_{\nu}}\left(t, x_{i}\right)\right) \leq \frac{y_{i}-x_{i}}{t}+\sqrt{\left\|\left(f^{\prime \prime}\right)-\right\|\left\|_{\infty}\right\| f^{\prime \prime} \|_{\infty}} \sum_{P \in \mathcal{D}\left(\bar{\gamma}_{i}^{x}\right) \cup \mathcal{D}\left(\bar{\gamma}_{i}^{y}\right)} \Delta T V(P)+\mathcal{O}\left(\varepsilon_{\nu}\right)
$$

in Case 1,

$$
f^{\prime}\left(u_{\varepsilon_{\nu}}\left(t, y_{i}\right)\right)-f^{\prime}\left(u_{\varepsilon_{\nu}}\left(t, x_{i}\right)\right) \leq\left[\dot{\gamma}_{i}^{y}\left(\tilde{t}_{i}^{+}\right)-\dot{\gamma}_{i}^{x}\left(\tilde{t}_{i}^{+}\right)\right]+\sqrt{\left\|\left(f^{\prime \prime}\right)-\right\|_{\infty}\left\|f^{\prime \prime}\right\|_{\infty}} \sum_{P \in \mathcal{D}\left(\bar{\gamma}_{i}^{x}\right) \cup \mathcal{D}\left(\bar{\gamma}_{i}^{y}\right)} \Delta T V(P)+\mathcal{O}\left(\varepsilon_{\nu}\right)
$$

in Case 2. (89)

Now there actually can be meetings of $\gamma_{i}^{x}$ and $\gamma_{j}^{y}(1 \leq i<j \leq n)$ for $t=\max \left(\tilde{t}_{i}, \tilde{t}_{j}\right)$ and the total variation dissipation at this point is used to measure the terms $\dot{\gamma}_{i}^{y}\left(\tilde{t}_{i}^{+}\right)-\dot{\gamma}_{i}^{x}\left(\tilde{t}_{i}^{+}\right)$appearing in (89). Suppose that such a meeting happens (call $\tilde{P}$ this interaction point): let us show that the total variation dissipation at the corresponding interaction point can be counted only once. Note that necessarily $\tilde{t}_{i}=\tilde{t}_{j}$ since, due to the assumption, $\gamma_{i}^{y}$ and $\gamma_{j}^{x}$ are between $\gamma_{i}^{x}$ and $\gamma_{j}^{y}$ for times $t>\max \left(\tilde{t}_{i}, \tilde{t}_{j}\right)$. We claim that, in place of $(67)$, stands

$$
\sum_{k \text { s.t. } \tilde{t}_{k}=\tilde{t}_{i}} \dot{\gamma}_{k}^{y}\left(t^{+}\right)-\dot{\gamma}_{k}^{x}\left(t^{+}\right) \leq \vartheta\left(u_{l}^{\tilde{P}} ; u_{r}^{\tilde{P}}\right)+\varepsilon_{\nu} \leq \sqrt{\left\|\left(f^{\prime \prime}\right)-\right\|_{\infty}\left\|f^{\prime \prime}\right\|_{\infty}} \Delta T V(\tilde{P})+3 \varepsilon_{\nu}
$$

The first inequality follows directly from the fact that the front paths $\gamma_{i}^{y}, \gamma_{k}^{x}, \gamma_{k}^{y}$ for $i<k<j$ such that $\tilde{t}_{k}=\tilde{t}_{i}$, and $\gamma_{j}^{x}$ are in the same order at time $\tilde{t}_{i}^{+}$as they are at time $T$ (they cannot change their order without crossing each other); the second one comes again from Proposition 4.

Now summing up the inequalities above yields (87) in the first case.

Second case. Let us assume that there is a meeting of $\gamma_{i}^{y}$ with $\gamma_{j}^{x}$ for a time $\bar{t}>\max \left(\tilde{t}_{i}, \tilde{t}_{j}\right)$, for some $1 \leq i<j \leq n$. We consider the largest time $\bar{t}$ for which this happens (among all times and indices $i, j$ ). If there is an ambiguity, consider $i$ the smallest index, and $j$ the largest index for which $\gamma_{i}^{y}$ meets $\gamma_{j}^{x}$ at time $\bar{t}$. Call $\bar{P}$ the interaction point at time $\bar{t}$. See for instance Figure 9 , where the curves $\gamma_{k}^{x}$ and $\gamma_{k}^{y}$ are represented only for $t \geq \tilde{t}_{k}$ (in that case $i=1$ and $j=4)$.

Now, we reduce $\mathcal{D}\left(\bar{\gamma}_{i}^{y}\right)$ and $\mathcal{D}\left(\bar{\gamma}_{j}^{x}\right)$ by removing the points in these sets corresponding to times $t \leq \bar{t}$. Note that $\gamma_{i}^{y}$ and $\gamma_{j}^{x}$ represent positive fronts at times $t \leq \bar{t}$ and immediately after $\bar{t}$ (since they meet). Consequently, the points that are removed belong to the paths $\gamma_{i}^{y}$ and $\gamma_{j}^{x}$.

Reasoning as previously and using the maximality of $\bar{t}$, one sees that $\mathcal{D}\left(\bar{\gamma}_{i}^{y}\right)$ and $\mathcal{D}\left(\bar{\gamma}_{j}^{x}\right)$ do no longer intersect other sets $\mathcal{D}$, nor other front paths $\gamma_{k}^{z}$, nor themselves. Moreover, due to the maximality of $\bar{t}$, for $k$ such that $i<k<j$ (if any), the front path $\gamma_{k}^{x}$ meets $\gamma_{k}^{y}$ at time $\tilde{t}_{k} \geq \bar{t}$ and do not cross other front paths $\gamma_{l}^{x}$ or $\gamma_{l}^{y}$ for $l=i, \ldots, k-1, k+1, \ldots, j$ for times $>\bar{t}$.

Now, we perform the same analysis as in Paragraph 4.4.2 to get

$$
\begin{aligned}
f^{\prime}\left(u_{\varepsilon_{\nu}}\left(y_{i}^{+}\right)\right)-f^{\prime}\left(u_{\varepsilon_{\nu}}\left(x_{j}^{-}\right)\right) & \leq \dot{\gamma}_{i}^{y}(T)-\dot{\gamma}_{j}^{x}(T)+3 \varepsilon_{\nu} \\
& \leq \dot{\gamma}_{i}^{y}(\bar{t})-\dot{\gamma}_{j}^{x}(\bar{t})+\sqrt{\left\|\left(f^{\prime \prime}\right)_{-}\right\|_{\infty}\left\|f^{\prime \prime}\right\|_{\infty}} \widetilde{\Delta} T V(T, x, y)+\mathcal{O}\left(\varepsilon_{\nu}\right) \\
& \leq \sqrt{\left\|\left(f^{\prime \prime}\right)_{-}\right\|_{\infty}\left\|f^{\prime \prime}\right\|_{\infty}} \widetilde{\Delta} T V(T, x, y)+\mathcal{O}\left(\varepsilon_{\nu}\right),
\end{aligned}
$$

where $\widetilde{\Delta} T V(T, x, y)$ is given again by (66), with the "new" sets $\mathcal{D}\left(\bar{\gamma}_{i}^{y}\right)$ and $\mathcal{D}\left(\bar{\gamma}_{j}^{x}\right)$. Note in particular that here we simply get $\dot{\gamma}_{i}^{y}(\bar{t})-\dot{\gamma}_{j}^{x}(\bar{t}) \leq 0$ from the fact that $\gamma_{i}^{y}$ is on the left of $\gamma_{j}^{x}$, and that we did not use the total 


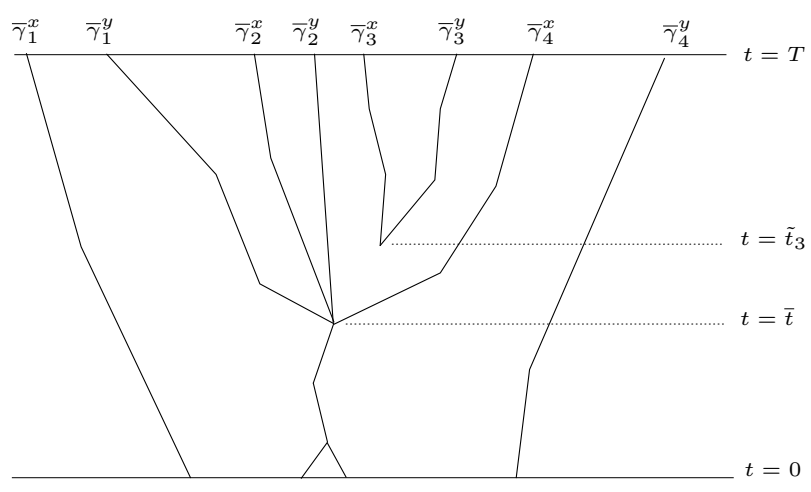

Figure 9: Crossing of $\gamma_{i}^{y}$ and $\gamma_{j}^{x}$

variation dissipation at $\bar{P}$ in the above estimate. Concerning the indices $k$ such that $i<k<j$, the above analysis of the first case applies, i.e. we can use (88)-(89)-(90). We get in total

$$
f^{\prime}\left(u_{\varepsilon_{\nu}}\left(y_{i}^{+}\right)\right)-f^{\prime}\left(u_{\varepsilon_{\nu}}\left(x_{j}^{-}\right)\right)+\sum_{i<k<j} f^{\prime}\left(u_{\varepsilon_{\nu}}\left(y_{k}^{+}\right)\right)-f^{\prime}\left(u_{\varepsilon_{\nu}}\left(x_{k}^{-}\right)\right) \leq \sqrt{\left\|\left(f^{\prime \prime}\right)-\right\|_{\infty}\left\|f^{\prime \prime}\right\|_{\infty}} \widehat{\Delta} T V(T, x, y)+\mathcal{O}\left(\varepsilon_{\nu}\right)
$$

with

$$
\widehat{\Delta} T V(T, x, y):=\Delta T V(\bar{P})+\sum_{(P, l) \in \mathcal{D}\left(\bar{\gamma}_{i}^{y}\right) \cup \mathcal{D}\left(\bar{\gamma}_{j}^{x}\right)} \Delta T V(P, l)+\sum_{\substack{(P, l) \in \mathcal{D}\left(\bar{\gamma}_{k}^{x}\right) \cup \mathcal{D}\left(\bar{\gamma}_{k}^{y}\right) \\ \text { for } i<k<j}} \Delta T V(P, l) .
$$

Now, we remove $y_{i}, x_{j}$ and all the intermediate points $x_{k}, y_{k}$ for $i<k<j$ from the list of the points $x_{1}, y_{1}, \ldots, x_{n}, y_{n}$. We now consider $\left(x_{i}, y_{j}\right)$ as the $i$-th couple, $\left(x_{j+1}, y_{j+1}\right)$ as the $(i+1)$-th couple if any etc., and reiterate the whole process. The various front paths $\gamma_{k}^{x}$ and $\gamma_{k}^{y}$ which are now selected are the same (except naturally those which have been removed), but now the numbering and the meeting times $\tilde{t}_{k}$ have changed. As a consequence, the points in the various sets $\mathcal{D}$ with negative outgoing fronts are the same, but there can be additional points in the sets $\mathcal{D}$ with positive outgoing fronts, located on the various front paths $\gamma_{k}^{z}$. Since the sets $\mathcal{D}$ which are used in (91)-(92) did no longer intersect other sets $\mathcal{D}$ nor other front paths $\gamma_{k}^{z}$, the result follows from an induction on $n$.

\subsection{Justification of Remark 1}

In this Paragraph, we briefly explain how one can obtain the constant of Remark 1.

It follows from the proof of Proposition 1 that the constant $\sqrt{\left\|\left(f^{\prime \prime}\right)_{-}\right\|_{\infty}\left\|f^{\prime \prime}\right\|_{\infty}}$ that we obtain is chosen as the worst constant between the one of Proposition 4 and the one of Lemma 3. Looking more closely to the proof of Proposition 4 (see in particular Lemma 8), we see that could have used the constant

$$
\max \left[\frac{\left\|\left(f^{\prime \prime}\right)-\right\|_{\infty}}{2}, \sqrt{\left\|\left(f^{\prime \prime}\right)-\right\|_{\infty}\left(\left\|\left(f^{\prime \prime}\right)-\right\|_{\infty}+\left\|\left(f^{\prime \prime}\right)_{+}\right\|_{\infty}\right)}-\left\|\left(f^{\prime \prime}\right)-\right\|_{\infty}\right]
$$

in Proposition 1.

Of course, the same remark for Proposition 2 yields the constant

$$
\max \left[\frac{\left\|\left(f^{\prime \prime}\right)_{+}\right\|_{\infty}}{2}, \sqrt{\left\|\left(f^{\prime \prime}\right)_{+}\right\|_{\infty}\left(\left\|\left(f^{\prime \prime}\right)_{+}\right\|_{\infty}+\left\|\left(f^{\prime \prime}\right)_{-}\right\|_{\infty}\right)}-\left\|\left(f^{\prime \prime}\right)_{+}\right\|_{\infty}\right]
$$

and as previously one takes the better of these two constants to get the main result.

\subsection{Proof of Theorem 2}

In this paragraph, we prove that one cannot replace $\sqrt{\left\|\left(f^{\prime \prime}\right)_{+}\right\|_{\infty}\left\|\left(f^{\prime \prime}\right)_{-}\right\|_{\infty}}$ with $\left\|\left(f^{\prime \prime}\right)_{-}\right\|_{\infty}$ (or even with $C\left\|\left(f^{\prime \prime}\right)_{-}\right\|_{\infty}$ for some constant $C$ independent from $\left.f\right)$ in (10). The same counterexample can easily be adapted to prove that one cannot replace this factor by $\left\|\left(f^{\prime \prime}\right)_{+}\right\|_{\infty}$ either.

The counterexample is given by a family of fluxes $\left(f_{n}\right)_{n \geq 1}$. These are constructed in the following way. Choose $\psi \in C^{\infty}\left(\mathbb{R}^{-}\right)$such that

$$
\left\{\begin{array}{l}
\psi=1 \text { on }(-\infty,-4], \\
\psi=0 \text { on }[-1,0], \\
\psi^{\prime} \leq 0 \text { on } \mathbb{R}^{-}, \\
\psi^{\prime \prime}<0 \text { on }(-4,-2), \\
\psi^{\prime \prime}>0 \text { on }(-2,-1) .
\end{array}\right.
$$


Such functions can easily be constructed. Now introduce $\Lambda \in C_{0}^{\infty}(0,1)$ satisfying

$$
\left\{\begin{array}{l}
\Lambda \geq 0 \\
\int \Lambda=1 / 2
\end{array}\right.
$$

Now for $n \geq 1, f_{n}$ is defined as

$$
f_{n}(x):=\left\{\begin{array}{l}
\Psi(x) \text { on } \mathbb{R}^{-} \\
\rho_{n}(x):=n \int_{0}^{x}\left(\int_{0}^{y} \Lambda((1-z) \sqrt{n}) d z\right) d y \text { on } \mathbb{R}^{+} .
\end{array}\right.
$$

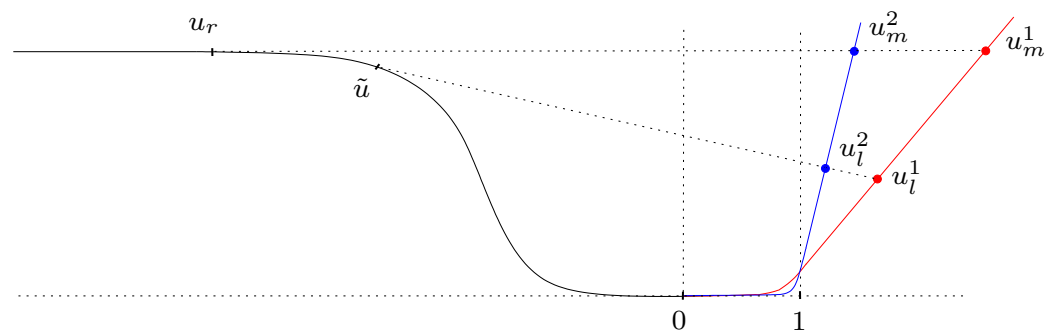

Figure 10: Counterexample: the fluxes

Note that $f_{n}(1) \leq 1 / 2$ for all $n \geq 1$, and that $f_{n}$ is affine for $x \geq 1$.

Now we define $u_{r}:=-4$ and we introduce $\tilde{u}>-4$ sufficiently close to -4 , in order that the tangent to the graph of $\Psi$ at $\tilde{u}$ cuts the axis $x=1$ at an ordinate greater than $1 / 2$. This is of course independent from $n$. For $n \in \mathbb{N}^{*}$, we define $u_{m}^{n}$ and $u_{l}^{n}$ in $\mathbb{R}^{+}$as solving the equations (see Figure 10):

$$
\rho_{n}\left(u_{m}^{n}\right):=1 \text { and } \Psi(\tilde{u})+\Psi^{\prime}(\tilde{u})\left(u_{l}^{n}-\tilde{u}\right)=\rho_{n}\left(u_{l}^{n}\right) .
$$

Clearly, $1<u_{l}^{n}<u_{m}^{n}$, and hence $f_{n}$ is affine in $\left[u_{l}^{n}, u_{m}^{n}\right]$.

Now consider, such as described in Figure 11, the meeting after some time of the right contact discontinuity $\left(u_{m}^{n} ; u_{r}\right)$ and the contact discontinuity $\left(u_{l}^{n} ; u_{m}^{n}\right)$.

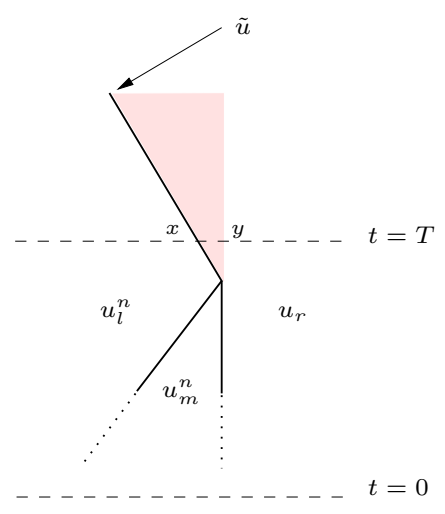

Figure 11: Counterexample: the interaction

This yields a left contact discontinuity $\left(u_{l}^{n} ; \tilde{u}\right)$ immediately followed by a rarefaction wave $\left(\tilde{u}, u_{r}\right)$ of constant inner speed variation. We choose $x$ and $y$ close enough to the interaction point such that $u(T, x)=\tilde{u}$ and $u(T, y)=u_{r}$, by taking the intersection of the sides of the outgoing wave fan with the line $t=T$, where $T$ is a time greater than the interaction time, but very close to it. Then we see that we arrive at a contradiction with

$$
f_{n}^{\prime}(u(T, y))-f_{n}^{\prime}(u(T, x)) \leq \frac{y-x}{T}+\left\|\left(f_{n}^{\prime \prime}\right)_{-}\right\|_{\infty}[T V(u(0, \cdot))-T V(u(T, \cdot))] \text { for } x \leq y \text { and } t>0,
$$

for $n$ large enough, because $\left\|\left(f_{n}^{\prime \prime}\right)_{-}\right\|_{\infty}$ and $f_{n}^{\prime}(u(t, y))-f_{n}^{\prime}(u(t, x))$ remain constant (positive for the latter), the term $\frac{y-x}{T}$ is negligible if $T$ is chosen sufficiently close the time of interaction (alternatively, we could choose the time of interaction large enough), but $T V(u(0, \cdot))-T V(u(t, \cdot))=u_{m}^{n}-u_{l}^{n}$ tends to 0 . 


\section{References}

[1] Bressan A., Hyperbolic systems of conservation laws, the one-dimensional problem, Oxford Lecture Series in Mathematics and its Applications 20. Oxford University Press, Oxford, 2000.

[2] Bressan A., Colombo R. M., Decay of positive waves in nonlinear systems of conservation laws, Ann. Sc. Norm. Sup. Pisa IV-26 (1998), pp. 133-160.

[3] Cheng K. S., A regularity theorem for a nonconvex scalar conservation law. J. Differential Equations 61 (1986), no. 1, pp. 79-127.

[4] Dafermos C. M., Polygonal approximations of solutions of the initial value problem for a conservation law. J. Math. Anal. Appl. 38 (1972), pp. 33-41.

[5] Dafermos C. M., Characteristics in hyperbolic conservation laws. A study of the structure and the asymptotic behaviour of solutions, Nonlinear analysis and mechanics: Heriot-Watt Symposium (Edinburgh, 1976), Vol. I, pp. 1-58. Res. Notes in Math., No. 17, Pitman, London, 1977.

[6] Dafermos C. M., Generalized characteristics and the structure of solutions of hyperbolic conservation laws. Indiana Univ. Math. J. 26 (1977), no. 6, pp. 1097-1119.

[7] Dafermos C. M., Hyperbolic conservation laws in continuum physics. Second edition. Grundlehren der Mathematischen Wissenschaften, 325. Springer-Verlag, Berlin, 2005.

[8] Glass O., LeFloch P. G., Nonlinear hyperbolic systems: non-degenerate flux, inner speed variation, and graph solutions, preprint 2006, to appear in Arch. Ration. Mech. Anal.

[9] Hoff D., The sharp form of Oleinik's entropy condition in several space variables. Trans. Amer. Math. Soc. 276 (1983), no. 2, pp. 707-714.

[10] Iguchi T., LeFloch P. G., Existence theory for hyperbolic systems of conservation laws with general fluxfunctions. Arch. Ration. Mech. Anal. 168 (2003), no. 3, pp. 165-244.

[11] Jenssen H. K., Sinestrari C., On the spreading of characteristics for non-convex conservation laws, Proc. Roy. Soc. Edinburgh Sect. A 131 (2001), no. 4, pp. 909-925.

[12] Kružkov S. N., First order quasilinear equations with several independent variables. (Russian) Mat. Sb. (N.S.) 81 (123) 1970, pp. 228-255. Translated in Math. USSR Sbornik Vol. 10 (1970), No. 2, pp. $217-243$.

[13] LeFloch P. G., Trivisa K., Continuous Glimm-type functionals and spreading of rarefaction waves, Commun. Math. Sci. 2 (2004), no. 2, pp. 213-236.

[14] Oleinik O. A., Discontinuous solutions of non-linear differential equations. Uspehi Mat. Nauk (N.S.) 12 (1957) no. 3(75), 3-73. (Translation in Ann. Math. Soc. Trans. Ser. 2 26, pp. 95-172). 